Groups Geom. Dyn. 4 (2010), 377-405

DOI $10.4171 / \mathrm{GGD} / 87$
Groups, Geometry, and Dynamics

(C) European Mathematical Society

\title{
On a series of finite automata defining free transformation groups
}

\author{
Mariya Vorobets and Yaroslav Vorobets*
}

\begin{abstract}
We introduce two series of finite automata starting from the so-called Aleshin and Bellaterra automata. We prove that transformations defined by automata from the first series generate a free non-Abelian group of infinite rank, while automata from the second series give rise to the free product of infinitely many groups of order 2 .
\end{abstract}

Mathematics Subject Classification (2010). 20E08, 20E05, $20 \mathrm{E} 06$.

Keywords. Automaton group, free non-Abelian group, finite automaton.

\section{Introduction}

A (Mealy) automaton over a finite alphabet $X$ is determined by the set of internal states, the state transition function and the output function. A finite (or finite-state) automaton has finitely many internal states. An initial automaton has a distinguished initial state. Any initial automaton over $X$ defines a transformation $T$ of the set $X^{*}$ of finite words in the alphabet $X$. That is, the automaton transduces any input word $w \in X^{*}$ into the output word $T(w)$. The transformation $T$ preserves the lengths of words and common beginnings. The set $X^{*}$ is endowed with the structure of a regular rooted tree so that $T$ is an endomorphism of the tree. A detailed account of the theory of Mealy automata is given in [GNS].

The set of all endomorphisms of the regular rooted tree $X^{*}$ is of continuum cardinality. Any endomorphism can be defined by an automaton. However the most interesting are finite automaton transformations that constitute a countable subset. If $T_{1}$ and $T_{2}$ are mappings defined by finite initial automata over the same alphabet $X$, then their composition is also defined by a finite automaton over $X$. If a finite automaton transformation $T$ is invertible, then the inverse transformation is also defined by a finite automaton. Furthermore, there are simple algorithms to construct the corresponding composition automaton and inverse automaton. In particular, all invertible

* Both authors partially supported by the NSF grants DMS-0308985 and DMS-0456185. The second author is supported by a Clay Research Scholarship. 
transformations defined by finite automata over $X$ constitute a transformation group $\mathscr{G}(X)$. This fact was probably first observed by Hořejš [Hoř].

A finite non-initial automaton $A$ over an alphabet $X$ defines a finite collection of transformations of $X^{*}$ corresponding to various choices of the initial state. Assuming all of them are invertible, these transformations generate a group $G(A)$, which is a finitely generated subgroup of $\mathscr{S}(X)$. We say that the group $G(A)$ is defined by the automaton $A$. The groups defined by finite automata were introduced by Grigorchuk [Gri] in connection with the Grigorchuk group of intermediate growth. The finite automaton nature of this group has great impact on its properties. The formalization of these properties has resulted in the notions of a branch group (see [BGS]), a fractal group (see $[\mathrm{BGN}]$ ), and, finally, the most general notion of a self-similar group [Nek], which covers all automaton groups.

The main issue of this paper are free non-Abelian groups of finite automaton transformations. Also, we are interested in the free products of groups of order 2 (such a product contains a free subgroup of index 2). Brunner and Sidki [BS] proved that the free group embeds into the group of finite automaton transformations over a 4-letter alphabet. Olijnyk [Oli1], [Oli2] showed that the group of finite automaton transformations over a 2-letter alphabet contains a free group as well as free products of groups of order 2. In the above examples, all automata are of linear algebraic origin.

A harder problem is to present the free group as the group defined by a single finite non-initial automaton. This problem was solved by Glasner and Mozes [GM]. They constructed infinitely many finite automata of algebraic origin that define transformation groups with various properties, in particular, free groups. A finite automaton that defines the free product of 3 groups of order 2 was found by Muntyan and Savchuk (see $[\mathrm{Nek}]$ and Theorem 1.5 below).

Actually, the first attempt to embed the free non-Abelian group into a group of finite automaton transformations was made by Aleshin [Ale] a long ago. He introduced two finite initial automata over alphabet $\{0,1\}$ and claimed that two automorphisms of the rooted binary tree $\{0,1\}^{*}$ defined by these automata generate a free group. However the argument in [Ale] seems to be incomplete. Aleshin's automata are depicted in Figure 1 by means of Moore diagrams. The Moore diagram of an automaton is a directed graph with labeled edges. The vertices are the states of the automaton and edges are state transition routes. Each label consists of two letters from the alphabet. The left one is the input field, it is used to choose a transition route. The right one is the output generated by the automaton. Aleshin considered these automata as initial, with initial state $b$.

The Aleshin automata are examples of bi-reversible automata. This notion, which generalizes the notion of invertibility, was introduced in [MNS] (see also [GM]). The class of bi-reversible automata is in a sense opposite to the class of automata defining branch groups. All automata considered in this paper are bi-reversible.

In this article we are looking for finite automata that define free non-Abelian groups of maximal rank, i.e., the free rank of the group is equal to the number of 

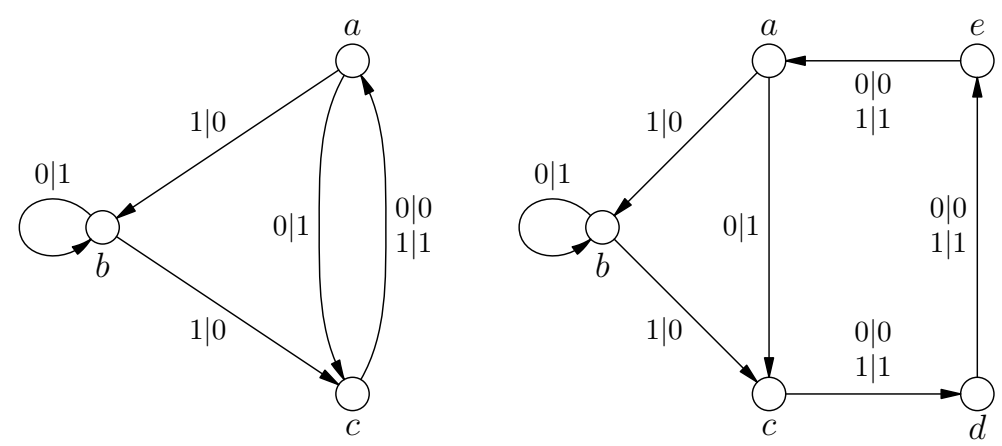

Figure 1. Aleshin's automata.

states of the automaton. Note that the automata constructed by Glasner and Mozes do not enjoy this property. For any of those automata, the transformations assigned to various internal states form a symmetric generating set so that the free rank of the group is half of the number of the states. Brunner and Sidki conjectured (see [Sid]) that the first of two Aleshin's automata shown in Figure 1 is the required one. The conjecture was proved in [VV].

Theorem 1.1 ([VV]). The first Aleshin automaton defines a free group on 3 generators.

In this article we generalize and extend Theorem 1.1 in several directions.

The two automata of Aleshin are related as follows. When the first automaton is in the state $c$, it is going to make transition to the state $a$ independently of the next input letter, which is sent directly to the output. The second automaton is obtained from the first one by inserting two additional states on the route from $c$ to $a$ (see Figure 1).

For any integer $n \geq 1$ we define a $(2 n+1)$-state automaton $A^{(n)}$ of Aleshin type. Up to renaming of internal states, $A^{(n)}$ is obtained from the first Aleshin automaton by inserting $2 n-2$ additional states on the route from $c$ to $a$ (for a precise definition, see Section 4); in particular, $A^{(1)}$ and $A^{(2)}$ are the Aleshin automata. The Moore diagram of the automaton $A^{(3)}$ is depicted in Figure 6 below. Note that the number of internal states of an Aleshin type automaton is always odd. This is crucial for the proof of the following theorem.

Theorem 1.2. Automorphisms of the rooted binary tree defined by automata of Aleshin type generate a free non-Abelian group of infinite rank. Moreover, all these automorphisms are free generators of the group.

In particular, any automaton of Aleshin type defines a free transformation group of maximal rank. 
Theorem 1.3. For any $n \geq 1$ the automaton $A^{(n)}$ defines a free group on $2 n+1$ generators.

Given a finite number of automata $Y^{(1)}, \ldots, Y^{(k)}$ over the same alphabet with disjoint sets of internal states $S_{1}, \ldots, S_{k}$, we can regard them as a single automaton $Y$ with the set of internal states $S_{1} \cup \cdots \cup S_{k}$. The automaton $Y$ is called the disjoint union of the automata $Y^{(1)}, \ldots, Y^{(k)}$ as its Moore diagram is the disjoint union of the Moore diagrams of $Y^{(1)}, \ldots, Y^{(k)}$. The group defined by $Y$ is generated by the groups $G\left(Y^{(1)}\right), \ldots, G\left(Y^{(k)}\right)$.

We define the Aleshin type automata so that their sets of internal states are disjoint. Hence the disjoint union of any finite number of distinct automata of Aleshin type is well defined. Clearly, Theorem 1.2 implies that such a disjoint union defines a free group of maximal rank.

Theorem 1.4. Let $N$ be a nonempty finite set of positive integers and denote by $A^{(N)}$ the disjoint union of automata $A^{(n)}, n \in N$. Then the automaton $A^{(N)}$ defines a free group on $\sum_{n \in N}(2 n+1)$ generators.

It is easy to see that Theorem 1.4 is actually equivalent to Theorem 1.2.

Another consequence of Theorem 1.2 is that the 8 transformations defined by the two Aleshin automata generate a free group on 8 generators. In particular, any two of them generate a free non-Abelian group. Thus Aleshin's claim is finally justified.

The Bellaterra automaton $B$ is a 3-state automaton over a 2-letter alphabet. Its Moore diagram is depicted in Figure 2. The automaton $B$ coincides with its inverse

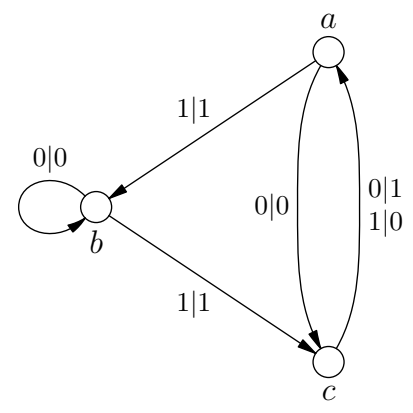

Figure 2. The Bellaterra automaton.

automaton and hence all 3 transformations defined by $B$ are involutions. Otherwise there are no more relations in the group $G(B)$.

Theorem 1.5 ([Nek]). The Bellaterra automaton defines the free product of 3 groups of order 2 . 
Theorem 1.5 is due to Muntyan and Savchuk. It was proved during the 2004 summer school on automata groups at the Autonomous University of Barcelona and so the automaton $B$ was named after the location of the university.

The Bellaterra automaton $B$ is closely related to the Aleshin automaton $A$. Namely, the two automata share the alphabet, internal states, and the state transition function while their output functions never coincide. We use this relation to define a series $B^{(1)}, B^{(2)}, \ldots$ of automata of Bellaterra type. By definition, $B^{(n)}$ is a $(2 n+1)$-state automaton obtained from $A^{(n)}$ by changing values of the output function at all elements of its domain. Also, we define a one-state automaton $B^{(0)}$ that interchanges letters 0 and 1 of the alphabet. All transformations defined by a Bellaterra type automaton are involutions.

Theorem 1.6. Automorphisms of the rooted binary tree defined by automata of Bellaterra type freely generate the free product of infinitely many groups of order 2 .

An equivalent, more detailed formulation of Theorem 1.6 is as follows.

Theorem 1.7. (i) For any $n \geq 0$ the automaton $B^{(n)}$ defines the free product of $2 n+1$ groups of order 2.

(ii) Let $N$ be a nonempty finite set of nonnegative integers and denote by $B^{(N)}$ the disjoint union of automata $B^{(n)}, n \in N$. Then the automaton $B^{(N)}$ defines the free product of $\sum_{n \in N}(2 n+1)$ groups of order 2 .

Theorems 1.4 and 1.7 have the following obvious corollary.

Corollary 1.8. (i) Let $n$ be an integer such that $n=3$ or $n=5$ or $n \geq 7$. Then there exists an $n$-state automaton over alphabet $\{0,1\}$ that define a free transformation group on $n$ generators.

(ii) For any integer $n \geq 3$ there exists an $n$-state automaton over alphabet $\{0,1\}$ that define a transformation group freely generated by $n$ involutions.

We prove Theorem 1.2 using the dual automaton approach. Namely, each finite automaton $Y$ is assigned a dual automaton $Y^{\prime}$ obtained from $Y$ by interchanging the alphabet with the set of internal states and the state transition function with the output function. It turns out that there is a connection between transformation groups defined by $Y$ and $Y^{\prime}$. As intermediate results, we obtain some information on the dual automata of the Aleshin type automata.

Proposition 1.9. (i) The dual automaton of the Aleshin automaton defines a group that acts transitively on each level of the rooted ternary tree $\{a, b, c\}^{*}$.

(ii) For any $n \geq 1$ the dual automaton of $A^{(n)}$ defines a group that acts transitively on each level of the rooted $(2 n+1)$-regular tree $Q_{n}^{*}$. 
The proof of Theorem 1.5 given in [Nek] also relies on the dual automaton approach. In particular, it involves a statement on the dual automaton $\widehat{D}$ of $B$. Since the group $G(B)$ is generated by involutions, it follows that the set of double letter words over the alphabet $\{a, b, c\}$ is invariant under the action of the group $G(\hat{D})$. Hence $G(\widehat{D})$ does not act transitively on levels of the rooted tree $\{a, b, c\}$.

Proposition 1.10 ([Nek]). The dual automaton of the Bellaterra automaton defines a transformation group that acts transitively on each level of the rooted subtree of $\{a, b, c\}^{*}$ formed by no-double-letter words.

We derive Theorem 1.6 from Theorem 1.2. This does not involve dual automata. Nonetheless we obtain a new proof of Proposition 1.10 that also works for all Bellaterra type automata.

Proposition 1.11. For any $n \geq 1$ the dual automaton of $B^{(n)}$ defines a group that acts transitively on each level of the rooted subtree of $Q_{n}^{*}$ formed by no-double-letter words.

Finally, we establish relations between groups defined by automata of Aleshin type and of Bellaterra type.

Proposition 1.12. (i) The group $G(A)$ is an index 2 subgroup of $G\left(B^{(\{0,1\})}\right)$.

(ii) For any $n \geq 1$ the group $G\left(A^{(n)}\right)$ is an index 2 subgroup of $G\left(B^{(\{0, n\})}\right)$.

(iii) For any nonempty finite set $N$ of positive integers the group $G\left(A^{(N)}\right)$ is an index 2 subgroup of $G\left(B^{(N \cup\{0\})}\right)$.

Proposition 1.13. (i) $G(A) \cap G(B)$ is a free group on 2 generators and an index 2 subgroup of $G(B)$.

(ii) For any $n \geq 1, G\left(A^{(n)}\right) \cap G\left(B^{(n)}\right)$ is a free group on $2 n$ generators and an index 2 subgroup of $G\left(B^{(n)}\right)$.

(iii) For any nonempty finite set $N$ of positive integers, $G\left(A^{(N)}\right) \cap G\left(B^{(N)}\right)$ is an index 2 subgroup of $G\left(B^{(N)}\right)$. Also, $G\left(A^{(N)}\right) \cap G\left(B^{(N)}\right)$ is a free group of rank less by 1 than the free rank of $G\left(A^{(N)}\right)$.

The article is organized as follows. Section 2 addresses some general constructions concerning automata and their properties. In Section 3 we recall constructions and arguments of the paper [VV] where Theorem 1.1 was proved. In Section 4 they are applied to the Aleshin type automata and their disjoint unions, which results in the proof of Theorem 1.4 (Theorem 4.10). Besides, Proposition 1.9 is established in Sections 3 and 4 (see Corollaries 3.6 and 4.9). Section 5 is devoted to the study of the Bellaterra automaton, automata of Bellaterra type, and their relation to automata of Aleshin type. Here we prove Theorems 1.5 and 1.7 (Theorems 5.3 and 5.4), Propositions 1.10 and 1.11 (Propositions 5.9 and 5.10), Proposition 1.12 (Proposition 5.2), and Proposition 1.13 (Propositions 5.6, 5.7, and 5.8). 


\section{Automata}

An automaton $A$ is a quadruple $(Q, X, \phi, \psi)$ formed by two nonempty sets $Q$ and $X$ along with two maps $\phi: Q \times X \rightarrow Q$ and $\psi: Q \times X \rightarrow X$. The set $X$ is to be finite, it is called the (input/output) alphabet of the automaton. We say that $A$ is an automaton over the alphabet $X . Q$ is called the set of internal states of $A$. The automaton $A$ is called finite (or finite-state) if the set $Q$ is finite. $\phi$ and $\psi$ are called the state transition function and the output function, respectively. One may regard these functions as a single map $(\phi, \psi): Q \times X \rightarrow Q \times X$.

The automaton $A$ canonically defines a collection of transformations. First we introduce the set on which these transformations act. This is the set of words over the alphabet $X$, which is denoted by $X^{*}$. A word $w \in X^{*}$ is merely a finite sequence whose elements belong to $X$. The elements of $w$ are called letters and $w$ is usually written so that its elements are not separated by delimiters. The number of letters of $w$ is called its length. It is assumed that $X^{*}$ contains the empty word $\varnothing$. The set $X$ is embedded in $X^{*}$ as the subset of one-letter words. If $w_{1}=x_{1} \ldots x_{n}$ and $w_{2}=y_{1} \ldots y_{m}$ are words over the alphabet $X$ then $w_{1} w_{2}$ denotes their concatenation $x_{1} \ldots x_{n} y_{1} \ldots y_{m}$. The operation $\left(w_{1}, w_{2}\right) \mapsto w_{1} w_{2}$ makes $X^{*}$ into the free monoid generated by all elements of $X$. The unit element of the monoid $X^{*}$ is the empty word. Another structure on $X^{*}$ is that of a rooted $k$-regular tree, where $k$ is the cardinality of $X$. Namely, we consider a graph with the set of vertices $X^{*}$ where two vertices $w_{1}, w_{2} \in X^{*}$ are joined by an edge if $w_{1}=w_{2} x$ or $w_{2}=w_{1} x$ for some $x \in X$. The root of the tree is the empty word. For any integer $n \geq 0$ the $n$-th level of a rooted tree is the set of vertices that are at distance $n$ from the root. Clearly, the $n$-th level of the rooted tree $X^{*}$ is formed by all words of length $n$ in the alphabet $X$.

Now let us explain how the automaton $A$ functions. First we choose an initial state $q \in Q$ and prepare an input word $w=x_{1} x_{2} \ldots x_{n} \in X^{*}$. Then we set the automaton to the state $q$ and start inputting the word $w$ into it, letter by letter. After reading a letter $x^{\prime}$ in a state $q^{\prime}$, the automaton produces the output letter $\psi\left(q^{\prime}, x^{\prime}\right)$ and makes transition to the state $\phi\left(q^{\prime}, x^{\prime}\right)$. Hence the automaton's job results in two sequences: a sequence of states $q_{0}=q, q_{1}, \ldots, q_{n}$, which describes the internal work of the automaton, and the output word $v=y_{1} y_{2} \ldots y_{n} \in X^{*}$. Here $q_{i}=\phi\left(q_{i-1}, x_{i}\right)$ and $y_{i}=\psi\left(q_{i-1}, x_{i}\right)$ for $1 \leq i \leq n$.

For every choice of the initial state $q \in Q$ of $A$ we get a mapping $A_{q}: X^{*} \rightarrow X^{*}$ that sends any input word to the corresponding output word. We say that $A_{q}$ is the transformation defined by the automaton $A$ with the initial state $q$. Clearly, $A_{q}$ preserves the length of words. Besides, $A_{q}$ transforms words from the left to the right, that is, the first $n$ letters of $A_{q}(w)$ depend only on the first $n$ letters of $w$. This implies that $A_{q}$ is an endomorphism of $X^{*}$ as a rooted tree. If $A_{q}$ is invertible then it belongs to the group $\operatorname{Aut}\left(X^{*}\right)$ of automorphisms of the rooted tree $X^{*}$. The set of transformations $A_{q}, q \in Q$ is self-similar in the following sense. For any $q \in Q, x \in X$, and $w \in X^{*}$ we have that $A_{q}(x w)=y A_{p}(w)$, where $p=\phi(q, x)$, $y=\psi(q, x)$. 
It must be noted that in many papers on automata the transformation $A_{q}$ is denoted simply $q$. However that short notation is not appropriate in our paper as we are going to consider different automata with the same set of states.

The semigroup of transformations of $X^{*}$ generated by $A_{q}, q \in Q$ is denoted by $S(A)$. The automaton $A$ is called invertible if $A_{q}$ is invertible for all $q \in Q$. If $A$ is invertible then $A_{q}, q \in Q$ generate a transformation group $G(A)$, which is a subgroup of $\operatorname{Aut}\left(X^{*}\right)$. We say that $S(A)$ (resp. $\left.G(A)\right)$ is the semigroup (resp. group) defined by the automaton $A$.

If $A$ is an invertible automaton then the restriction of any transformation $g \in G(A)$ to a level of the rooted tree $X^{*}$ is a permutation on this finite set. As a consequence, the restriction of the semigroup $S(A)$ to any level of the tree is actually a group (while the semigroup itself need not be a group). This implies that the actions of $S(A)$ and $G(A)$ on $X^{*}$ have the same orbits.

One way to picture an automaton, which we use in this paper, is the Moore diagram. The Moore diagram of an automaton $A=(Q, X, \phi, \psi)$ is a directed graph with labeled edges defined as follows. The vertices of the graph are states of the automaton $A$. Every edge carries a label of the form $x \mid y$, where $x, y \in X$. The left field $x$ of the label is referred to as the input field while the right field $y$ is referred to as the output field. The set of edges of the graph is in a one-to-one correspondence with the set $Q \times X$. Namely, for any $q \in Q$ and $x \in X$ there is an edge that goes from the vertex $q$ to $\phi(q, x)$ and carries the label $x \mid \psi(q, x)$. The Moore diagram of an automaton can have loops (edges joining a vertex to itself) and multiple edges. To simplify pictures, we do not draw multiple edges in this paper. Instead, we use multiple labels.

The transformations $A_{q}, q \in Q$ can be defined in terms of the Moore diagram of the automaton $A$. For any $q \in Q$ and $w \in X^{*}$ we find a path $\gamma$ in the Moore diagram such that $\gamma$ starts at the vertex $q$ and the word $w$ can be obtained by reading the input fields of labels along $\gamma$. Such a path exists and is unique. Then the word $A_{q}(w)$ is obtained by reading the output fields of labels along the path $\gamma$.

Let $\Gamma$ denote the Moore diagram of the automaton $A$. We associate to $\Gamma$ two directed graphs $\Gamma_{1}$ and $\Gamma_{2}$ with labeled edges. $\Gamma_{1}$ is obtained from $\Gamma$ by interchanging the input and output fields of all labels. That is, a label $x \mid y$ is replaced by $y \mid x . \Gamma_{2}$ is obtained from $\Gamma$ by reversing all edges. The inverse automaton of $A$ is the automaton whose Moore diagram is $\Gamma_{1}$. The reverse automaton of $A$ is the automaton whose Moore diagram is $\Gamma_{2}$. The inverse and reverse automata of $A$ share the alphabet and internal states with $A$. Notice that any automaton is completely determined by its Moore diagram. However neither $\Gamma_{1}$ nor $\Gamma_{2}$ must be the Moore diagram of an automaton. So it is possible that the inverse automaton or the reverse automaton (or both) of $A$ is not well defined.

Lemma 2.1 ([GNS]). An automaton $A=(Q, X, \phi, \psi)$ is invertible if and only if for any $q \in Q$ the map $\psi(q, \cdot): X \rightarrow X$ is bijective. The inverse automaton I of $A$ is well defined if and only if $A$ is invertible. In this case, $I_{q}=A_{q}^{-1}$ for all $q \in Q$. 
An automaton $A$ is called reversible if the reverse automaton of $A$ is well defined.

Lemma 2.2 ([VV]). An automaton $A=(Q, X, \phi, \psi)$ is reversible if and only if for any $x \in X$ the map $\phi(\cdot, x): Q \rightarrow Q$ is bijective.

Let $A=(Q, X, \phi, \psi)$ be an automaton. Let $A_{\xi}=A_{q_{n}} \ldots A_{q_{2}} A_{q_{1}}$ for any nonempty word $\xi=q_{1} q_{2} \ldots q_{n} \in Q^{*}$. Set $A_{\varnothing}=1$ (here 1 stands for the unit element of the group $\operatorname{Aut}\left(X^{*}\right)$, i.e., the identity mapping on $\left.X^{*}\right)$. Clearly, any element of the semigroup $S(A)$ is represented as $A_{\xi}$ for a nonempty word $\xi \in Q^{*}$. The map $X^{*} \times Q^{*} \rightarrow X^{*}$ given by $(w, \xi) \mapsto A_{\xi}(w)$ defines a right action of the monoid $Q^{*}$ on the rooted regular tree $X^{*}$. That is, $A_{\xi_{1} \xi_{2}}(w)=A_{\xi_{2}}\left(A_{\xi_{1}}(w)\right)$ for all $\xi_{1}, \xi_{2} \in Q^{*}$ and $w \in X^{*}$.

To each finite automaton $A=(Q, X, \phi, \psi)$ we associate a dual automaton $D$, which is obtained from $A$ by interchanging the alphabet with the set of internal states and the state transition function with the output function. To be precise, $D=$ $(X, Q, \widetilde{\phi}, \widetilde{\psi})$, where $\widetilde{\phi}(x, q)=\psi(q, x)$ and $\widetilde{\psi}(x, q)=\phi(q, x)$ for all $x \in X$ and $q \in Q$. Unlike the inverse and reverse automata, the dual automaton is always well defined. It is easy to see that $A$ is the dual automaton of $D$.

The dual automaton $D$ defines a right action of the monoid $X^{*}$ on $Q^{*}$ given by $(\xi, w) \mapsto D_{w}(\xi)$. This action and the action of $Q^{*}$ on $X^{*}$ defined by the automaton $A$ are related in the following way.

Proposition 2.3 ([VV]). For any $w, u \in X^{*}$ and $\xi \in Q^{*}$,

$$
A_{\xi}(w u)=A_{\xi}(w) A_{D_{w}(\xi)}(u) .
$$

Corollary 2.4 ([VV]). Suppose that $A_{\xi}=1$ for some $\xi \in Q^{*}$. Then $A_{g(\xi)}=1$ for every $g \in S(D)$.

A finite automaton $A=(Q, X, \phi, \psi)$ is called bi-reversible if the map $\phi(\cdot, x): Q \rightarrow Q$ is bijective for any $x \in X$, the map $\psi(q, \cdot): X \rightarrow X$ is bijective for any $q \in Q$, and the map $(\phi, \psi): Q \times X \rightarrow Q \times X$ is bijective as well. All automata that we consider in this paper are bi-reversible. Below we formulate some basic properties of bi-reversible automata (see also [Nek]).

Lemma 2.5. Given a finite automaton A, the following are equivalent:

(i) $A$ is bi-reversible.

(ii) $A$ is invertible, reversible, and its reverse automaton is invertible.

(iii) $A$ is invertible, reversible, and its inverse automaton is reversible.

(iv) A is invertible, its dual automaton is invertible, and the dual automaton of its inverse is invertible.

Proof. Suppose that $A=(Q, X, \phi, \psi)$ is a finite automaton. By Lemma 2.1, $A$ is invertible if and only if maps $\psi(q, \cdot): X \rightarrow X$ are bijective for all $q \in Q$. By 
Lemma 2.2, $A$ is reversible if and only if maps $\phi(\cdot, x): Q \rightarrow Q$ are bijective for all $x \in X$. Let $\Gamma$ be the Moore diagram of $A$ and $\Gamma^{\prime}$ be the graph obtained from $\Gamma$ by reversing all edges and interchanging fields of all labels. The graph $\Gamma^{\prime}$ is the Moore diagram of an automaton if for any $q \in Q$ and $x \in X$ there is exactly one edge of $\Gamma^{\prime}$ that starts at the vertex $q$ and has $x$ as the input field of its label. By definition of $\Gamma^{\prime}$ the number of edges with the latter property is equal to the number of pairs $(p, y) \in Q \times X$ such that $q=\phi(p, y)$ and $x=\psi(p, y)$. Therefore $\Gamma^{\prime}$ is the Moore diagram of an automaton if and only if the map $(\phi, \psi): Q \times X \rightarrow Q \times X$ is bijective. Thus $A$ is bi-reversible if and only if it is invertible, reversible, and $\Gamma^{\prime}$ is the Moore diagram of an automaton.

Assume that the automaton $A$ is invertible and reversible. Let $I$ and $R$ be the inverse and reverse automata of $A$, respectively. If the graph $\Gamma^{\prime}$ is the Moore diagram of an automaton then the automaton is both the inverse automaton of $R$ and the reverse automaton of $I$. On the other hand, if $\Gamma^{\prime}$ is not the Moore diagram of an automaton then $R$ is not invertible and $I$ is not reversible. It follows that conditions (i), (ii), and (iii) are equivalent.

It follows from Lemmas 2.1 and 2.2 that a finite automaton is reversible if and only if its dual automaton is invertible. This implies that conditions (iii) and (iv) are equivalent.

Lemma 2.6. If an automaton is bi-reversible then its inverse, reverse, and dual automata are also bi-reversible.

Proof. It follows directly from definitions that an automaton is bi-reversible if and only if its dual automaton is bi-reversible.

Suppose that $A$ is a bi-reversible automaton. By Lemma 2.5, $A$ is invertible and reversible. Let $I$ and $R$ denote the inverse and reverse automata of $A$, respectively. By Lemma 2.5, $I$ is reversible and $R$ is invertible. It is easy to see that $A$ is both the inverse automaton of $I$ and the reverse automaton of $R$. Therefore the automata $I$ and $R$ are invertible and reversible. Moreover, the inverse automaton of $I$ is reversible and the reverse automaton of $R$ is invertible. By Lemma 2.5, the automata $I$ and $R$ are bi-reversible.

Suppose that $A^{(1)}=\left(Q_{1}, X, \phi_{1}, \psi_{1}\right), \ldots, A^{(k)}=\left(Q_{k}, X, \phi_{k}, \psi_{k}\right)$ are automata over the same alphabet $X$ such that their sets of internal states $Q_{1}, Q_{2}, \ldots, Q_{k}$ are disjoint. The disjoint union of automata $A^{(1)}, A^{(2)}, \ldots, A^{(k)}$ is an automaton $U=$ $\left(Q_{1} \cup \cdots \cup Q_{k}, X, \phi, \psi\right)$, where the functions $\phi, \psi$ are defined so that $\phi=\phi_{i}$ and $\psi=\psi_{i}$ on $Q_{i} \times X$ for $1 \leq i \leq k$. Obviously, $U_{q}=A_{q}^{(i)}$ for all $q \in Q_{i}, 1 \leq i \leq k$. The Moore diagram of the automaton $U$ is the disjoint union of the Moore diagrams of $A^{(1)}, A^{(2)}, \ldots, A^{(k)}$. This observation easily leads to the following statement.

Lemma 2.7. The disjoint union of automata $A^{(1)}, A^{(2)}, \ldots, A^{(k)}$ is invertible (resp.reversible, bi-reversible) if and only if each $A^{(i)}$ is invertible (resp. reversible, bireversible). 


\section{The Aleshin automaton}

In this section we recall constructions and results of the paper [VV] where the Aleshin automaton was studied. Some constructions are slightly modified.

The Aleshin automaton is an automaton $A$ over the alphabet $X=\{0,1\}$ with the set of internal states $Q=\{a, b, c\}$. The state transition function $\phi$ and the output function $\psi$ of $A$ are defined as follows: $\phi(a, 0)=\phi(b, 1)=c, \phi(a, 1)=\phi(b, 0)=$ $b, \phi(c, 0)=\phi(c, 1)=a ; \psi(a, 0)=\psi(b, 0)=\psi(c, 1)=1, \psi(a, 1)=\psi(b, 1)=$ $\psi(c, 0)=0$. The Moore diagram of $A$ is depicted in Figure 1. It is easy to verify that the automaton $A$ is invertible and reversible. Moreover, the inverse automaton of $A$ can be obtained from $A$ by renaming letters 0 and 1 of the alphabet to 1 and 0 , respectively. The reverse automaton of $A$ can be obtained from $A$ by renaming its states $a$ and $c$ to $c$ and $a$, respectively. Lemma 2.5 implies that $A$ is bi-reversible.
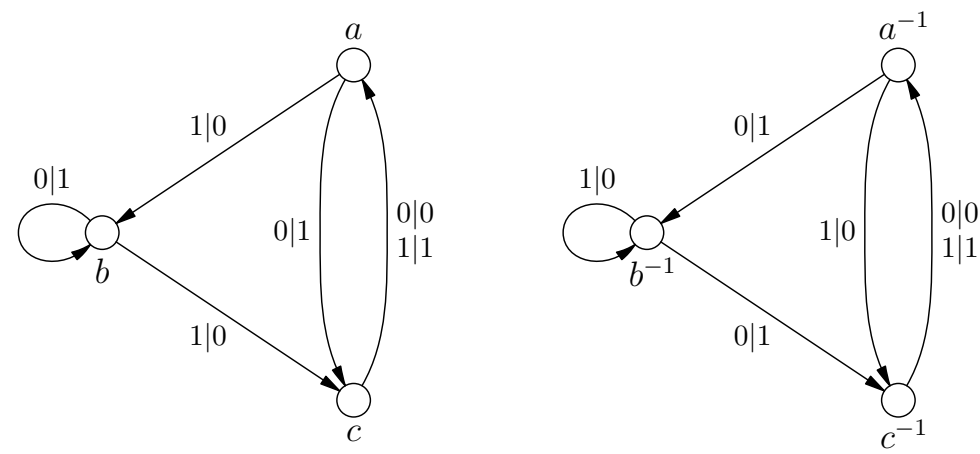

Figure 3. Automaton $U$.

Let $I$ denote the automaton obtained from the inverse of $A$ by renaming its states $a, b, c$ to $a^{-1}, b^{-1}, c^{-1}$, respectively. Here, $a^{-1}, b^{-1}$, and $c^{-1}$ are assumed to be elements of the free group on generators $a, b, c$. Further, let $U$ denote the disjoint union of automata $A$ and $I$. The automaton $U$ is defined over the alphabet $X=$ $\{0,1\}$, with the set of internal states $Q^{ \pm}=\left\{a, b, c, a^{-1}, b^{-1}, c^{-1}\right\}$. By definition and Lemma 2.1, $U_{a}=A_{a}, U_{b}=A_{b}, U_{c}=A_{c}, U_{a^{-1}}=A_{a}^{-1}, U_{b^{-1}}=A_{b}^{-1}$, $U_{c^{-1}}=A_{c}^{-1}$.

Let $D$ denote the dual automaton of the automaton $U$. The automaton $D$ is defined over the alphabet $Q^{ \pm}$, with two internal states 0 and 1 . By $\phi_{D}$ denote its transition function. Then $\phi_{D}(0, q)=1$ and $\phi_{D}(1, q)=0$ for $q \in\left\{a, b, a^{-1}, b^{-1}\right\}$, while $\phi_{D}(0, q)=0$ and $\phi_{D}(1, q)=1$ for $q \in\left\{c, c^{-1}\right\}$. Also, we consider an auxiliary automaton $E$ that is closely related to $D$. By definition, the automaton $E$ shares with $D$ the alphabet, the set of internal states, and the state transition function. The output function $\psi_{E}$ of $E$ is defined so that $\psi_{E}(0, q)=\sigma_{0}(q)$ and $\psi_{E}(1, q)=\sigma_{1}(q)$ for all $q \in Q^{ \pm}$, where $\sigma_{0}=\left(a^{-1} b^{-1}\right)$ and $\sigma_{1}=(a b)$ are permutations on the set $Q^{ \pm}$. 


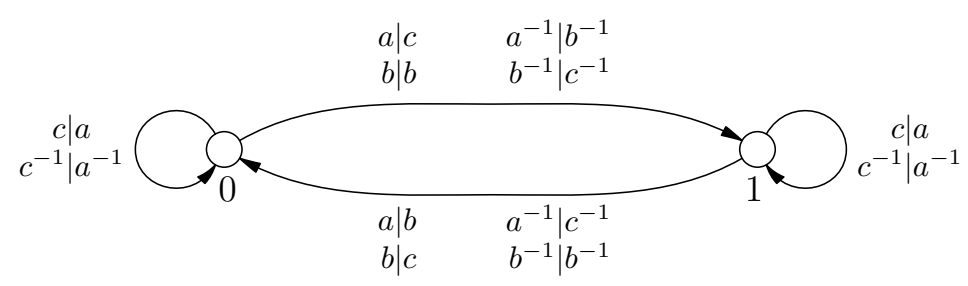

Figure 4. Automaton $D$.

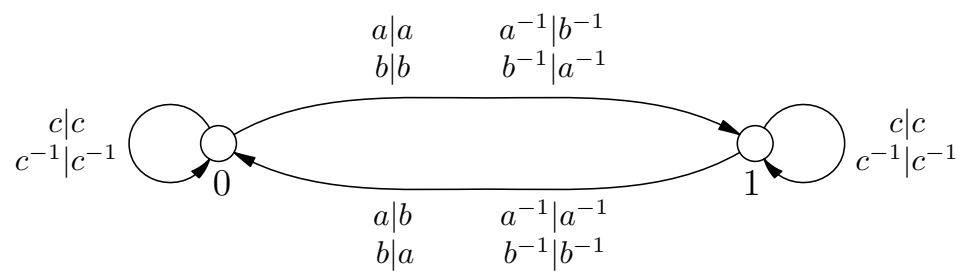

Figure 5. Automaton $E$.

Lemmas 2.6 and 2.7 imply that $I, U$, and $D$ are bi-reversible automata. As for the automaton $E$, it is easy to verify that $E$ coincides with its inverse automaton while the reverse automaton of $E$ can be obtained from $E$ by renaming its states 0 and 1 to 1 and 0 , respectively. Hence $E$ is bi-reversible due to Lemma 2.5.

To each permutation $\tau$ on the set $Q=\{a, b, c\}$ we assign an automorphism $\pi_{\tau}$ of the free monoid $\left(Q^{ \pm}\right)^{*}$. The automorphism $\pi_{\tau}$ is uniquely defined by $\pi_{\tau}(q)=\tau(q)$, $\pi_{\tau}\left(q^{-1}\right)=(\tau(q))^{-1}$ for all $q \in Q$. Let $\langle a, b, c\rangle$ denote the free group on generators $a, b$, and $c$, let $\delta:\left(Q^{ \pm}\right)^{*} \rightarrow\langle a, b, c\rangle$ be the homomorphism that sends each element of $Q^{ \pm} \subset\left(Q^{ \pm}\right)^{*}$ to itself, and let $p_{\tau}$ be the automorphism of $\langle a, b, c\rangle$ defined by $p_{\tau}(q)=\tau(q), q \in Q$. Then $\delta\left(\pi_{\tau}(\xi)\right)=p_{\tau}(\delta(\xi))$ for all $\xi \in\left(Q^{ \pm}\right)^{*}$.

Lemma 3.1 ([VV]). (i) $E_{0}^{2}=E_{1}^{2}=1, E_{0} E_{1}=E_{1} E_{0}=\pi_{(a b)}$.

(ii) $D_{0}=\pi_{(a c)} E_{0}=\pi_{(a b c)} E_{1}, D_{1}=\pi_{(a b c)} E_{0}=\pi_{(a c)} E_{1}$.

Proposition 3.2 ([VV]). The group $G(D)$ contains $E_{0}, E_{1}$, and all transformations of the form $\pi_{\tau}$. Moreover, $G(D)$ is generated by $E_{0}, \pi_{(a b)}$, and $\pi_{(b c)}$.

As described in Section 2, the automaton $U$ defines a right action $X^{*} \times\left(Q^{ \pm}\right)^{*} \rightarrow$ $X^{*}$ of the monoid $\left(Q^{ \pm}\right)^{*}$ on the rooted binary tree $X^{*}$ given by $(w, \xi) \mapsto U_{\xi}(w)$. Let $\chi:\left(Q^{ \pm}\right)^{*} \rightarrow\{-1,1\}$ be the unique homomorphism such that $\chi(a)=\chi(b)=$ $\chi\left(a^{-1}\right)=\chi\left(b^{-1}\right)=-1, \chi(c)=\chi\left(c^{-1}\right)=1$.

Lemma 3.3 ([VV]). Given $\xi \in\left(Q^{ \pm}\right)^{*}$, the automorphism $U_{\xi}$ of the rooted binary tree $\{0,1\}^{*}$ acts trivially on the first level of the tree (i.e., on one-letter words) if and only if $\chi(\xi)=1$. 
Now we introduce an alphabet consisting of two symbols $*$ and $*^{-1}$. A word over the alphabet $\left\{*, *^{-1}\right\}$ is called a pattern. Every word $\xi$ over the alphabet $Q^{ \pm}$is assigned a pattern $v$ that is obtained from $\xi$ by substituting $*$ for each occurrence of letters $a, b, c$ and substituting $*^{-1}$ for each occurrence of letters $a^{-1}, b^{-1}, c^{-1}$. We say that $v$ is the pattern of $\xi$ or that $\xi$ follows the pattern $v$.

A word $\xi=q_{1} q_{2} \ldots q_{n} \in\left(Q^{ \pm}\right)^{*}$ is called freely irreducible if none of its twoletter subwords $q_{1} q_{2}, q_{2} q_{3}, \ldots, q_{n-1} q_{n}$ coincides with one of the following words: $a a^{-1}, b b^{-1}, c c^{-1}, a^{-1} a, b^{-1} b, c^{-1} c$. Otherwise $\xi$ is called freely reducible.

Lemma 3.4 ([VV]). For any nonempty pattern $v$ there exist words $\xi_{1}, \xi_{2} \in\left(Q^{ \pm}\right)^{*}$ such that $\xi_{1}$ and $\xi_{2}$ are freely irreducible, follow the pattern $v$, and $\chi\left(\xi_{2}\right)=-\chi\left(\xi_{1}\right)$.

Proposition 3.5 ([VV]). Suppose that $\xi \in\left(Q^{ \pm}\right)^{*}$ is a freely irreducible word. Then the orbit of $\xi$ under the action of the group $G(D)$ on $\left(Q^{ \pm}\right)^{*}$ consists of all freely irreducible words following the same pattern as $\xi$.

Corollary 3.6. The group defined by the dual automaton of A acts transitively on each level of the rooted ternary tree $Q^{*}$.

Proof. Let $D^{+}$denote the dual automaton of $A$. The rooted tree $Q^{*}$ is a subtree of $\left(Q^{ \pm}\right)^{*}$. It is easy to see that $Q^{*}$ is invariant under transformations $D_{0}, D_{1}$ and the restrictions of these transformations to $Q^{*}$ are $D_{0}^{+}, D_{1}^{+}$. In particular, the orbits of the $G\left(D^{+}\right)$action on $Q^{*}$ are those orbits of the $G(D)$ action on $\left(Q^{ \pm}\right)^{*}$ that are contained in $Q^{*}$. Any level of the tree $Q^{*}$ consists of words of a fixed length over the alphabet $Q$. As elements of $\left(Q^{ \pm}\right)^{*}$, all these words are freely irreducible and follow the same pattern. Proposition 3.5 implies that they are in the same orbit of the $G\left(D^{+}\right)$action.

Lemmas 3.3, 3.4 and Proposition 3.5 lead to the following statement.

Theorem 3.7 ([VV]). The group $G(A)$ is the free non-Abelian group on generators $A_{a}, A_{b}, A_{c}$.

\section{Series of finite automata of Aleshin type}

In this section we consider a series of finite automata starting from the Aleshin automaton. Also, we consider disjoint unions of such automata. We use the notation of the previous section.

For any integer $n \geq 1$ we define an Aleshin type automaton $A^{(n)}$. This is an automaton over the alphabet $X=\{0,1\}$ with a set of states $Q_{n}$ of cardinality $2 n+1$. The states of $A^{(n)}$ are denoted so that $Q_{1}=\left\{a_{1}, b_{1}, c_{1}\right\}$ and $Q_{n}=$ $\left\{a_{n}, b_{n}, c_{n}, q_{n 1}, \ldots, q_{n, 2 n-2}\right\}$ for $n \geq 2$. The state transition function $\phi_{n}$ of $A^{(n)}$ is defined as follows: $\phi_{n}\left(a_{n}, 0\right)=\phi_{n}\left(b_{n}, 1\right)=c_{n}, \phi_{n}\left(a_{n}, 1\right)=\phi_{n}\left(b_{n}, 0\right)=b_{n}$, 
and $\phi_{n}\left(q_{n i}, 0\right)=\phi_{n}\left(q_{n i}, 1\right)=q_{n, i+1}$ for $0 \leq i \leq 2 n-2$, where by definition $q_{n 0}=c_{n}$ and $q_{n, 2 n-1}=a_{n}$. The output function $\psi_{n}$ of $A^{(n)}$ is defined so that for any $x \in X$ we have $\psi_{n}(q, x)=1-x$ if $q \in\left\{a_{n}, b_{n}\right\}$ and $\psi_{n}(q, x)=x$ if $q \in Q_{n} \backslash\left\{a_{n}, b_{n}\right\}$.

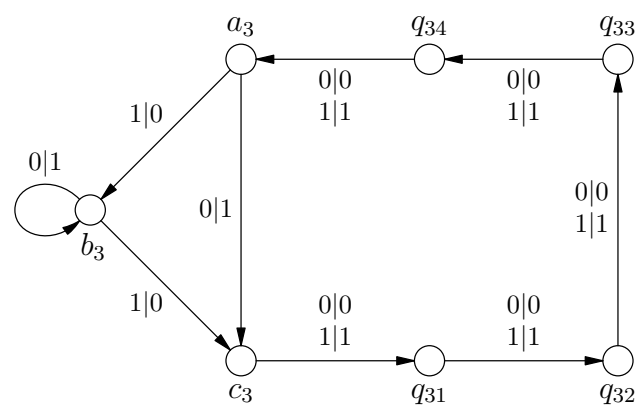

Figure 6. Automaton $A^{(3)}$.

Up to renaming of the internal states, $A^{(1)}$ and $A^{(2)}$ are the two automata introduced by Aleshin [Ale] (see Figure 1).

We shall deal with automata $A^{(n)}$ by following the framework developed in the paper [VV] and described in Section 3.

Let us fix a positive integer $n$. It is easy to see that the inverse automaton of the automaton $A^{(n)}$ can be obtained from $A^{(n)}$ by renaming letters 0 and 1 of the alphabet to 1 and 0 , respectively. Besides, the reverse automaton of $A^{(n)}$ can be obtained from $A^{(n)}$ by renaming its states $c_{n}, q_{n 1}, \ldots, q_{n, 2 n-2}, a_{n}$ to $a_{n}, q_{n, 2 n-2}, \ldots, q_{n 1}, c_{n}$, respectively. Lemma 2.5 implies that $A^{(n)}$ is bi-reversible.

Let $I^{(n)}$ denote the automaton obtained from the inverse of $A^{(n)}$ by renaming each state $q \in Q_{n}$ to $q^{-1}$, where $q^{-1}$ is regarded as an element of the free group on generators $a_{n}, b_{n}, c_{n}, q_{n 1}, \ldots, q_{n, 2 n-2}$. Now that the states of the inverse automaton are renamed, we can form the disjoint union $U^{(n)}$ of automata $A^{(n)}$ and $I^{(n)}$. The automaton $U^{(n)}$ is defined over the alphabet $X=\{0,1\}$, with the set of internal states $Q_{n}^{ \pm}=\bigcup_{q \in Q_{n}}\left\{q, q^{-1}\right\}$. By definition and Lemma 2.1, $U_{q}^{(n)}=A_{q}^{(n)}$ and $U_{q^{-1}}^{(n)}=\left(A_{q}^{(n)}\right)^{-1}$ for all $q \in Q_{n}$.

Let $D^{(n)}$ denote the dual automaton of the automaton $U^{(n)}$. The automaton $D^{(n)}$ is defined over the alphabet $Q_{n}^{ \pm}$, with two internal states 0 and 1 . By $\lambda_{n}$ denote its transition function. Then $\lambda_{n}(0, q)=1$ and $\lambda_{n}(1, q)=0$ if $q \in\left\{a_{n}, b_{n}, a_{n}^{-1}, b_{n}^{-1}\right\}$, while $\lambda_{n}(0, q)=0$ and $\lambda_{n}(1, q)=1$ otherwise. Also, we consider an auxiliary automaton $E^{(n)}$. By definition, the automaton $E^{(n)}$ shares with $D^{(n)}$ the alphabet, the set of internal states, and the state transition function. The output function $\mu_{n}$ of $E^{(n)}$ is defined so that $\mu_{n}(0, q)=\sigma_{0}(q)$ and $\mu_{n}(1, q)=\sigma_{1}(q)$ for all $q \in Q_{n}^{ \pm}$, where $\sigma_{0}=\left(a_{n}^{-1} b_{n}^{-1}\right)$ and $\sigma_{1}=\left(a_{n} b_{n}\right)$ are permutations on the set $Q_{n}^{ \pm}$. 
Lemmas 2.6 and 2.7 imply that $I^{(n)}, U^{(n)}$, and $D^{(n)}$ are bi-reversible automata. Further, it is easy to see that the automaton $E^{(n)}$ coincides with its inverse automaton while the reverse automaton of $E^{(n)}$ can be obtained from $E^{(n)}$ by renaming its states 0 and 1 to 1 and 0 , respectively. By Lemma $2.5, E^{(n)}$ is bi-reversible.

To each permutation $\tau$ on the set $Q_{n}$ we assign an automorphism $\pi_{\tau}^{(n)}$ of the free monoid $\left(Q_{n}^{ \pm}\right)^{*}$ such that $\pi_{\tau}^{(n)}(q)=\tau(q), \pi_{\tau}^{(n)}\left(q^{-1}\right)=(\tau(q))^{-1}$ for all $q \in Q_{n}$. The automorphism $\pi_{\tau}^{(n)}$ is uniquely determined by $\tau$.

Lemma 4.1. (i) $\left(E_{0}^{(n)}\right)^{2}=\left(E_{1}^{(n)}\right)^{2}=1, E_{0}^{(n)} E_{1}^{(n)}=E_{1}^{(n)} E_{0}^{(n)}=\pi_{\left(a_{n} b_{n}\right)}^{(n)}$.

(ii) $D_{0}^{(n)}=\pi_{\tau_{0}}^{(n)} E_{0}^{(n)}=\pi_{\tau_{1}}^{(n)} E_{1}^{(n)}, D_{1}^{(n)}=\pi_{\tau_{1}}^{(n)} E_{0}^{(n)}=\pi_{\tau_{0}}^{(n)} E_{1}^{(n)}$, where $\tau_{0}=$ $\left(a_{n} c_{n} q_{n 1} \ldots q_{n, 2 n-2}\right), \tau_{1}=\left(a_{n} b_{n} c_{n} q_{n 1} \ldots q_{n, 2 n-2}\right)$.

Proof. Since the inverse automaton of $E^{(n)}$ coincides with $E^{(n)}$, Lemma 2.1 implies that $\left(E_{0}^{(n)}\right)^{2}=\left(E_{1}^{(n)}\right)^{2}=1$.

We have that $E^{(n)}=\left(X, Q_{n}^{ \pm}, \lambda_{n}, \mu_{n}\right)$, where the functions $\lambda_{n}$ and $\mu_{n}$ are defined above. Note that the function $\lambda_{n}$ does not change when elements 0 and 1 of the set $X$ are renamed to 1 and 0 , respectively. For any permutation $\sigma$ on the set $Q_{n}^{ \pm}$we define an automaton $Y^{\sigma}=\left(X, Q_{n}^{ \pm}, \lambda_{n}, \sigma \mu_{n}\right)$. The Moore diagram of $Y^{\sigma}$ is obtained from the Moore diagram of $E^{(n)}$ by applying $\sigma$ to the output fields of all labels. It is easy to observe that $Y_{0}^{\tau}=\alpha_{\sigma} E_{0}^{(n)}$ and $Y_{1}^{\tau}=\alpha_{\sigma} E_{1}^{(n)}$, where $\alpha_{\sigma}$ is the unique automorphism of the monoid $\left(Q_{n}^{ \pm}\right)^{*}$ such that $\alpha_{\sigma}(q)=\sigma(q)$ for all $q \in Q_{n}^{ \pm}$.

Let us consider the following permutations on $Q_{n}^{ \pm}$:

$$
\begin{aligned}
& \sigma_{0}=\left(a_{n}^{-1} b_{n}^{-1}\right), \\
& \sigma_{1}=\left(a_{n} b_{n}\right), \\
& \sigma_{2}=\left(a_{n} b_{n}\right)\left(a_{n}^{-1} b_{n}^{-1}\right), \\
& \sigma_{3}=\left(a_{n} c_{n} q_{n 1} \ldots q_{n, 2 n-2}\right)\left(a_{n}^{-1} b_{n}^{-1} c_{n}^{-1} q_{n 1}^{-1} \ldots q_{n, 2 n-2}^{-1}\right), \\
& \sigma_{4}=\left(a_{n} b_{n} c_{n} q_{n 1} \ldots q_{n, 2 n-2}\right)\left(a_{n}^{-1} c_{n}^{-1} q_{n 1}^{-1} \ldots q_{n, 2 n-2}^{-1}\right), \\
& \sigma_{5}=\left(a_{n} c_{n} q_{n 1} \ldots q_{n, 2 n-2}\right)\left(a_{n}^{-1} c_{n}^{-1} q_{n 1}^{-1} \ldots q_{n, 2 n-2}^{-1}\right), \\
& \sigma_{6}=\left(a_{n} b_{n} c_{n} q_{n 1} \ldots q_{n, 2 n-2}\right)\left(a_{n}^{-1} b_{n}^{-1} c_{n}^{-1} q_{n 1}^{-1} \ldots q_{n, 2 n-2}^{-1}\right) .
\end{aligned}
$$

Since $\sigma_{2} \sigma_{0}=\sigma_{1}$ and $\sigma_{2} \sigma_{1}=\sigma_{0}$, it follows that the automaton $Y^{\sigma_{2}}$ can be obtained from $E^{(n)}$ by renaming its states 0 and 1 to 1 and 0 , respectively. Therefore $E_{0}^{(n)}=Y_{1}^{\sigma_{2}}=\alpha_{\sigma_{2}} E_{1}^{(n)}$ and $E_{1}^{(n)}=Y_{0}^{\sigma_{2}}=\alpha_{\sigma_{2}} E_{0}^{(n)}$. Consequently, $E_{0}^{(n)} E_{1}^{(n)}=$ $\alpha_{\sigma_{2}}\left(E_{1}^{(n)}\right)^{2}=\alpha_{\sigma_{2}}$ and $E_{1}^{(n)} E_{0}^{(n)}=\alpha_{\sigma_{2}}\left(E_{0}^{(n)}\right)^{2}=\alpha_{\sigma_{2}}$. Clearly, $\alpha_{\sigma_{2}}=\pi_{\left(a_{n} b_{n}\right)}^{(n)}$.

Since $\sigma_{5} \sigma_{0}=\sigma_{3}$ and $\sigma_{5} \sigma_{1}=\sigma_{4}$, it follows that $Y^{\sigma_{5}}=D^{(n)}$. Hence $D_{0}^{(n)}=$ $\alpha_{\sigma_{5}} E_{0}^{(n)}$ and $D_{1}^{(n)}=\alpha_{\sigma_{5}} E_{1}^{(n)}$. Furthermore, the equalities $\sigma_{6} \sigma_{0}=\sigma_{4}$ and $\sigma_{6} \sigma_{1}=\sigma_{3}$ imply that the automaton $Y^{\sigma_{6}}$ can be obtained from $D^{(n)}$ by renaming its states 0 and 1 to 1 and 0 , respectively. Therefore $D_{0}^{(n)}=Y_{1}^{\sigma_{6}}=\alpha_{\sigma_{6}} E_{1}^{(n)}$ and $D_{1}^{(n)}=Y_{0}^{\sigma_{6}}=$ $\alpha_{\sigma_{6}} E_{0}^{(n)}$. It remains to notice that $\alpha_{\sigma_{5}}=\pi_{\tau_{0}}^{(n)}$ and $\alpha_{\sigma_{6}}=\pi_{\tau_{1}}^{(n)}$. 
Proposition 4.2. The group $G\left(D^{(n)}\right)$ contains $E_{0}^{(n)}, E_{1}^{(n)}$, and all transformations of the form $\pi_{\tau}^{(n)}$. Moreover, $G\left(D^{(n)}\right)$ is generated by $E_{0}^{(n)}, \pi_{\tau_{0}}^{(n)}$, and $\pi_{\tau_{1}}^{(n)}$, where $\tau_{0}=\left(a_{n} c_{n} q_{n 1} \ldots q_{n, 2 n-2}\right), \tau_{1}=\left(a_{n} b_{n} c_{n} q_{n 1} \ldots q_{n, 2 n-2}\right)$.

Proof. It is easy to see that $\pi_{\tau \sigma}^{(n)}=\pi_{\tau}^{(n)} \pi_{\sigma}^{(n)}$ for any permutations $\tau$ and $\sigma$ on the set $Q_{n}$. It follows that $\pi_{\tau^{-1}}^{(n)}=\left(\pi_{\tau}^{(n)}\right)^{-1}$ for any permutation $\tau$ on $Q_{n}$.

By Lemma 4.1, the group generated by $E_{0}^{(n)}, \pi_{\tau_{0}}^{(n)}$, and $\pi_{\tau_{1}}^{(n)}$ contains $G\left(D^{(n)}\right)$. Besides, $D_{0}^{(n)}\left(D_{1}^{(n)}\right)^{-1}=\pi_{\tau_{0}}^{(n)} E_{0}^{(n)}\left(\pi_{\tau_{1}}^{(n)} E_{0}^{(n)}\right)^{-1}=\pi_{\tau_{0}}^{(n)}\left(\pi_{\tau_{1}}^{(n)}\right)^{-1}$. By the above $\pi_{\tau_{0}}^{(n)}\left(\pi_{\tau_{1}}^{(n)}\right)^{-1}=\pi_{\tau_{2}}^{(n)}$, where $\tau_{2}=\tau_{0} \tau_{1}^{-1}=\left(b_{n} c_{n}\right)$. Similarly,

$$
\left(D_{0}^{(n)}\right)^{-1} D_{1}^{(n)}=\left(\pi_{\tau_{0}}^{(n)} E_{0}^{(n)}\right)^{-1} \pi_{\tau_{1}}^{(n)} E_{0}^{(n)}=\left(E_{0}^{(n)}\right)^{-1} \pi_{\tau_{3}}^{(n)} E_{0}^{(n)},
$$

where $\tau_{3}=\tau_{0}^{-1} \tau_{1}=\left(a_{n} b_{n}\right)$. Lemma 4.1 implies that $E_{0}^{(n)}$ and $\pi_{\tau_{3}}^{(n)}$ commute, hence $\left(D_{0}^{(n)}\right)^{-1} D_{1}^{(n)}=\pi_{\tau_{3}}^{(n)}$. Consider two more permutations on $Q_{n}: \tau_{4}=\left(a_{n} c_{n}\right)$ and $\tau_{5}=\left(c_{n} q_{n 1} \ldots q_{n, 2 n-2}\right)$. Note that $\tau_{4}=\tau_{2} \tau_{3} \tau_{2}$ and $\tau_{5}=\tau_{4} \tau_{0}$. By the above $\pi_{\tau_{2}}^{(n)}, \pi_{\tau_{3}}^{(n)} \in G\left(D^{(n)}\right)$, hence $\pi_{\tau_{4}}^{(n)} \in G\left(D^{(n)}\right)$. Then $\pi_{\tau_{5}}^{(n)} E_{0}^{(n)}=\pi_{\tau_{4}}^{(n)} \pi_{\tau_{0}}^{(n)} E_{0}^{(n)}=$ $\pi_{\tau_{4}}^{(n)} D_{0}^{(n)} \in G\left(D^{(n)}\right)$. Since $\tau_{5}\left(a_{n}\right)=a_{n}$ and $\tau_{5}\left(b_{n}\right)=b_{n}$, it easily follows that transformations $\pi_{\tau_{5}}^{(n)}$ and $E_{0}^{(n)}$ commute. As $\tau_{5}$ is a permutation of odd order $2 n-1$ while $E_{0}^{(n)}$ is an involution, we have that $\left(\pi_{\tau_{5}}^{(n)} E_{0}^{(n)}\right)^{2 n-1}=E_{0}^{(n)}$. In particular, $E_{0}^{(n)} \in G\left(D^{(n)}\right)$. Now Lemma 4.1 implies that $\pi_{\tau_{0}}^{(n)}, \pi_{\tau_{1}}^{(n)}, E_{1}^{(n)} \in G\left(D^{(n)}\right)$.

It is easy to observe that the group of all permutations on the set $Q_{n}$ is generated by permutations $\tau_{1}=\left(a_{n} b_{n} c_{n} q_{n 1} \ldots q_{n, 2 n-2}\right)$ and $\tau_{3}=\left(a_{n} b_{n}\right)$. Since $\pi_{\tau_{1}}^{(n)}, \pi_{\tau_{3}}^{(n)} \in$ $G\left(D^{(n)}\right)$, it follows that $G\left(D^{(n)}\right)$ contains all transformations of the form $\pi_{\tau}^{(n)}$.

Now we proceed to disjoint unions of Aleshin type automata. Let $N$ be a nonempty finite set of positive integers. We denote by $A^{(N)}$ the disjoint union of automata $A^{(n)}$, $n \in N$. Then $A^{(N)}$ is an automaton over the alphabet $X=\{0,1\}$ with the set of internal states $Q_{N}=\bigcup_{n \in N} Q_{n}$. It is bi-reversible since each $A^{(n)}$ is bi-reversible. If the set $N$ consists of a single integer $n$ then $A^{(N)}=A^{(n)}$.

Let $I^{(N)}$ denote the disjoint union of automata $I^{(n)}, n \in N$. The automaton $I^{(N)}$ can be obtained from the inverse of $A^{(N)}$ by renaming each state $q \in Q_{N}$ to $q^{-1}$. Further, let $U^{(N)}$ denote the disjoint union of automata $A^{(N)}$ and $I^{(N)}$. Obviously, the automaton $U^{(N)}$ is also the disjoint union of automata $U^{(n)}, n \in N$. It is defined over the alphabet $X=\{0,1\}$, with the set of internal states $Q_{N}^{ \pm}=\bigcup_{n \in N} Q_{n}^{ \pm}$. Clearly, $U_{q}^{(N)}=A_{q}^{(N)}$ and $U_{q^{-1}}^{(N)}=\left(A_{q}^{(N)}\right)^{-1}$ for all $q \in Q_{N}$

Let $D^{(N)}$ denote the dual automaton of the automaton $U^{(N)}$. The automaton $D^{(N)}$ is defined over the alphabet $Q_{N}^{ \pm}$, with two internal states 0 and 1. Also, we consider an auxiliary automaton $E^{(N)}$. By definition, the automaton $E^{(N)}$ shares with $D^{(N)}$ the alphabet, the set of internal states, and the state transition function. The output function $\mu_{N}$ of $E^{(N)}$ is defined so that $\mu_{N}(0, q)=\sigma_{0}(q)$ and $\mu_{N}(1, q)=\sigma_{1}(q)$ for 
all $q \in Q_{N}^{ \pm}$, where $\sigma_{0}=\prod_{n \in N}\left(a_{n}^{-1} b_{n}^{-1}\right)$ and $\sigma_{1}=\prod_{n \in N}\left(a_{n} b_{n}\right)$ are permutations on the set $Q_{N}^{ \pm}$.

Lemmas 2.6 and 2.7 imply that $I^{(N)}, U^{(N)}$, and $D^{(N)}$ are bi-reversible automata. Further, it is easy to see that the automaton $E^{(N)}$ coincides with its inverse automaton while the reverse automaton of $E^{(N)}$ can be obtained from $E^{(N)}$ by renaming its states 0 and 1 to 1 and 0 , respectively. By Lemma $2.5, E^{(N)}$ is bi-reversible.

To each permutation $\tau$ on the set $Q_{N}$ we assign an automorphism $\pi_{\tau}^{(N)}$ of the free monoid $\left(Q_{N}^{ \pm}\right)^{*}$ such that $\pi_{\tau}^{(N)}(q)=\tau(q), \pi_{\tau}^{(N)}\left(q^{-1}\right)=(\tau(q))^{-1}$ for all $q \in Q_{N}$. The automorphism $\pi_{\tau}^{(N)}$ is uniquely determined by $\tau$.

Lemma 4.3. (i) $\left(E_{0}^{(N)}\right)^{2}=\left(E_{1}^{(N)}\right)^{2}=1, E_{0}^{(N)} E_{1}^{(N)}=E_{1}^{(N)} E_{0}^{(N)}=\pi_{\tau}^{(N)}$, where $\tau=\prod_{n \in N}\left(a_{n} b_{n}\right)$.

(ii) $D_{0}^{(N)}=\pi_{\tau_{0}}^{(N)} E_{0}^{(N)}=\pi_{\tau_{1}}^{(N)} E_{1}^{(N)}, D_{1}^{(N)}=\pi_{\tau_{1}}^{(N)} E_{0}^{(N)}=\pi_{\tau_{0}}^{(N)} E_{1}^{(N)}$, where $\tau_{0}=\prod_{n \in N}\left(a_{n} c_{n} q_{n 1} \ldots q_{n, 2 n-2}\right), \tau_{1}=\prod_{n \in N}\left(a_{n} b_{n} c_{n} q_{n 1} \ldots q_{n, 2 n-2}\right)$.

The proof of Lemma 4.3 is completely analogous to the proof of Lemma 4.1 and we omit it.

Proposition 4.4. The group $G\left(D^{(N)}\right)$ contains transformations $E_{0}^{(N)}, E_{1}^{(N)}, \pi_{\tau_{1}}^{(N)}$, $\pi_{\tau_{2}}^{(N)}, \pi_{\tau_{3}}^{(N)}$, and $\pi_{\tau_{4}}^{(N)}$, where $\tau_{1}=\prod_{n \in N}\left(a_{n} b_{n} c_{n} q_{n 1} \ldots q_{n, 2 n-2}\right), \tau_{2}=$ $\prod_{n \in N}\left(c_{n} q_{n 1} \ldots q_{n, 2 n-2}\right), \tau_{3}=\prod_{n \in N}\left(a_{n} b_{n}\right)$, and $\tau_{4}=\prod_{n \in N}\left(b_{n} c_{n}\right)$.

Proof. We follow the proof of Lemma 4.2. First let us notice that $\pi_{\tau \sigma}^{(N)}=\pi_{\tau}^{(N)} \pi_{\sigma}^{(N)}$ and $\pi_{\tau^{-1}}^{(N)}=\left(\pi_{\tau}^{(N)}\right)^{-1}$ for any permutations $\tau$ and $\sigma$ on the set $Q_{N}$.

By Lemma 4.3, $D_{0}^{(N)}\left(D_{1}^{(N)}\right)^{-1}=\pi_{\tau_{0}}^{(N)} E_{0}^{(N)}\left(\pi_{\tau_{1}}^{(N)} E_{0}^{(N)}\right)^{-1}=\pi_{\tau_{0}}^{(N)}\left(\pi_{\tau_{1}}^{(N)}\right)^{-1}$, where $\tau_{0}=\prod_{n \in N}\left(a_{n} c_{n} q_{n 1} \ldots q_{n, 2 n-2}\right)$. In view of $\tau_{0} \tau_{1}^{-1}=\tau_{4}$ it follows that $D_{0}^{(N)}\left(D_{1}^{(N)}\right)^{-1}=\pi_{\tau_{4}}^{(N)}$. Similarly,

$$
\left(D_{0}^{(N)}\right)^{-1} D_{1}^{(N)}=\left(\pi_{\tau_{0}}^{(N)} E_{0}^{(N)}\right)^{-1} \pi_{\tau_{1}}^{(N)} E_{0}^{(N)}=\left(E_{0}^{(N)}\right)^{-1} \pi_{\tau_{3}}^{(N)} E_{0}^{(N)}
$$

since $\tau_{3}=\tau_{0}^{-1} \tau_{1}$. Lemma 4.3 implies that $E_{0}^{(N)}$ and $\pi_{\tau_{3}}^{(N)}$ commute. Therefore $\left(D_{0}^{(N)}\right)^{-1} D_{1}^{(N)}=\pi_{\tau_{3}}^{(N)}$. Consider the permutation $\tau_{5}=\prod_{n \in N}\left(a_{n} c_{n}\right)$ on $Q_{N}$. Notice that $\tau_{5}=\tau_{4} \tau_{3} \tau_{4}$ and $\tau_{2}=\tau_{5} \tau_{0}$. By the above $\pi_{\tau_{3}}^{(N)}, \pi_{\tau_{4}}^{(N)} \in G\left(D^{(N)}\right)$, hence $\pi_{\tau_{5}}^{(N)} \in G\left(D^{(N)}\right)$. Then $\pi_{\tau_{2}}^{(N)} E_{0}^{(N)}=\pi_{\tau_{5}}^{(N)} \pi_{\tau_{0}}^{(N)} E_{0}^{(N)}=\pi_{\tau_{5}}^{(N)} D_{0}^{(N)} \in$ $G\left(D^{(N)}\right)$. Since $\tau_{2}\left(a_{n}\right)=a_{n}$ and $\tau_{2}\left(b_{n}\right)=b_{n}$ for all $n \in N$, it easily follows that transformations $\pi_{\tau_{2}}^{(N)}$ and $E_{0}^{(N)}$ commute. As $\tau_{2}$ is the product of commuting permutations of odd orders $2 n-1, n \in N$, while $E_{0}^{(N)}$ is an involution, we have that $\left(\pi_{\tau_{2}}^{(N)} E_{0}^{(N)}\right)^{m}=E_{0}^{(N)}$, where $m=\prod_{n \in N}(2 n-1)$. In particular, $E_{0}^{(N)}$ and $\pi_{\tau_{2}}^{(N)}$ are contained in $G\left(D^{(N)}\right)$. Now Lemma 4.3 implies that $\pi_{\tau_{1}}^{(N)}, E_{1}^{(N)} \in G\left(D^{(N)}\right)$. 
Recall that words over the alphabet $\left\{*, *^{-1}\right\}$ are called patterns. Every word $\xi \in\left(Q_{N}^{ \pm}\right)^{*}$ is assigned a pattern $v$ that is obtained from $\xi$ by substituting $*$ for each occurrence of letters $q \in Q_{N}$ and substituting $*^{-1}$ for each occurrence of letters $q^{-1}$, $q \in Q_{N}$. We say that $\xi$ follows the pattern $v$.

Now we introduce an alphabet $P_{N}^{ \pm}$that consists of symbols $*_{n}$ and $*_{n}^{-1}$ for all $n \in N$. A word over the alphabet $P_{N}^{ \pm}$is called a marked pattern. Every word $\xi \in\left(Q_{N}^{ \pm}\right)^{*}$ is assigned a marked pattern $v \in\left(P_{N}^{ \pm}\right)^{*}$ that is obtained from $\xi$ as follows. For any $n \in N$ we substitute $*_{n}$ for each occurrence of letters $q \in Q_{n}$ in $\xi$ and substitute $*_{n}^{-1}$ for each occurrence of letters $q^{-1}, q \in Q_{n}$. We say that $\xi$ follows the marked pattern $v$. Clearly, the pattern of $\xi$ is uniquely determined by its marked pattern. Notice that each letter of the alphabet $P_{N}^{ \pm}$corresponds to a connected component of the Moore diagram of the automaton $U^{(N)}$. Since $D^{(N)}$ is the dual automaton of $U^{(N)}$, it easily follows that the $G\left(D^{(N)}\right)$ action on $\left(Q_{N}^{ \pm}\right)^{*}$ preserves marked patterns of words.

A word $\xi=q_{1} q_{2} \ldots q_{k} \in\left(Q_{N}^{ \pm}\right)^{*}$ is called freely irreducible if none of its twoletter subwords $q_{1} q_{2}, q_{2} q_{3}, \ldots, q_{k-1} q_{k}$ is of the form $q q^{-1}$ or $q^{-1} q$, where $q \in Q_{N}$. Otherwise $\xi$ is called freely reducible.

Lemma 4.5. For any nonempty word $v \in\left(P_{N}^{ \pm}\right)^{*}$ there exists a freely irreducible word $\xi \in\left(Q_{N}^{ \pm}\right)^{*}$ such that $v$ is the marked pattern of $\xi$ and the transformation $U_{\xi}^{(N)}$ acts nontrivially on the first level of the rooted binary tree $X^{*}$.

Proof. For any $n \in N$ let us substitute $a_{n}$ for each occurrence of $*_{n}$ in $v$ and $b_{n}^{-1}$ for each occurrence of $*_{n}^{-1}$. We get a nonempty word $\xi \in\left(Q_{N}^{ \pm}\right)^{*}$ that follows the marked pattern $v$. Now let us modify $\xi$ by changing its last letter. If this letter is $a_{n}$ ( $n \in N$ ), we change it to $c_{n}$. If the last letter of $\xi$ is $b_{n}^{-1}$, we change it to $c_{n}^{-1}$. This yields another word $\eta \in\left(Q_{N}^{ \pm}\right)^{*}$ that follows the marked pattern $v$. By construction, $\xi$ and $\eta$ are freely irreducible. Furthermore, $U_{\eta}^{(N)}=A_{c_{n}}^{(n)}\left(A_{a_{n}}^{(n)}\right)^{-1} U_{\xi}^{(N)}$ if the last letter of $v$ is $*_{n}, n \in N$, while $U_{\eta}^{(N)}=\left(A_{c_{n}}^{(n)}\right)^{-1} A_{b_{n}}^{(n)} U_{\xi}^{(N)}$ if the last letter of $v$ is $*_{n}^{-1}$. For any $n \in N$ both $A_{c_{n}}^{(n)}\left(A_{a_{n}}^{(n)}\right)^{-1}$ and $\left(A_{c_{n}}^{(n)}\right)^{-1} A_{b_{n}}^{(n)}$ interchange one-letter words 0 and 1. It follows that one of the transformations $U_{\xi}^{(N)}$ and $U_{\eta}^{(N)}$ also acts nontrivially on the first level of the rooted tree $\{0,1\}^{*}$.

Given a nonempty, freely irreducible word $\xi \in\left(Q_{N}^{ \pm}\right)^{*}$, let $Z_{N}(\xi)$ denote the set of all freely irreducible words in $\left(Q_{N}^{ \pm}\right)^{*}$ that follow the same marked pattern as $\xi$ and match $\xi$ completely or except for the last letter. Obviously, $\xi \in Z_{N}(\xi)$, and $\eta \in Z_{N}(\xi)$ if and only if $\xi \in Z_{N}(\eta)$.

Lemma 4.6. For any nonempty word $v \in\left(P_{N}^{ \pm}\right)^{*}$ there exists a freely irreducible word $\xi \in\left(Q_{N}^{ \pm}\right)^{*}$ such that $v$ is the marked pattern of $\xi$ an the set $Z_{N}(\xi)$ is contained in one orbit of the $G\left(D^{(N)}\right)$ action on $\left(Q_{N}^{ \pm}\right)^{*}$. 
Proof. Let $\widetilde{Q}_{N}^{ \pm}=\bigcup_{n \in N}\left\{a_{n}, b_{n}, c_{n}, a_{n}^{-1}, b_{n}^{-1}, c_{n}^{-1}\right\}$. The set $\left(\widetilde{Q}_{N}^{ \pm}\right)^{*}$ of words in the alphabet $\widetilde{Q}_{N}^{ \pm}$is a submonoid of $\left(Q_{N}^{ \pm}\right)^{*}$. Let $h_{N}:\left(\widetilde{Q}_{N}^{ \pm}\right)^{*} \rightarrow\left(Q^{ \pm}\right)^{*}$ be the homomorphism of monoids such that $h_{N}\left(a_{n}\right)=a, h_{N}\left(b_{n}\right)=b, h_{N}\left(c_{n}\right)=c$, $h_{N}\left(a_{n}^{-1}\right)=a^{-1}, h_{N}\left(b_{n}^{-1}\right)=b^{-1}, h_{N}\left(c_{n}^{-1}\right)=c^{-1}$ for all $n \in N$. For any $\zeta \in\left(\widetilde{Q}_{N}^{ \pm}\right)^{*}$ the word $h_{N}(\zeta)$ follows the same pattern as $\zeta$. The word $\zeta$ is uniquely determined by $h_{N}(\zeta)$ and the marked pattern of $\zeta$. If $h_{N}(\zeta)$ is freely irreducible then so is $\zeta$ (however $h_{N}(\zeta)$ can be freely reducible even if $\zeta$ is freely irreducible). It is easy to see that $E_{0}\left(h_{N}(\zeta)\right)=h_{N}\left(E_{0}^{(N)}(\zeta)\right), \pi_{(a b)}\left(h_{N}(\zeta)\right)=h_{N}\left(\pi_{\sigma_{1}}^{(N)}(\zeta)\right)$, and $\pi_{(b c)}\left(h_{N}(\zeta)\right)=h_{N}\left(\pi_{\sigma_{2}}^{(N)}(\zeta)\right)$, where $\sigma_{1}=\prod_{n \in N}\left(a_{n} b_{n}\right)$ and $\sigma_{2}=\prod_{n \in N}\left(b_{n} c_{n}\right)$ are permutations on $Q_{N}$. By Proposition 3.2, the group $G(D)$ is generated by $E_{0}, \pi_{(a b)}$, and $\pi_{(b c)}$. On the other hand, $E_{0}^{(N)}, \pi_{\sigma_{1}}^{(N)}, \pi_{\sigma_{2}}^{(N)} \in G\left(D^{(N)}\right)$ due to Proposition 4.4. Let $\widetilde{G}$ denote the subgroup of $G\left(D^{(N)}\right)$ generated by $E_{0}^{(N)}, \pi_{\sigma_{1}}^{(N)}$, and $\pi_{\sigma_{2}}^{(N)}$. It follows that for any $g_{0} \in G(D)$ there exists $g \in \widetilde{G}$ such that $g_{0}\left(h_{N}(\zeta)\right)=h_{N}(g(\zeta))$ for all $\zeta \in\left(\widetilde{Q}_{N}^{ \pm}\right)^{*}$. Now Proposition 3.5 implies that words $\zeta_{1}, \zeta_{2} \in\left(\widetilde{Q}_{N}^{ \pm}\right)^{*}$ are in the same orbit of the $G\left(D^{(N)}\right)$ action on $\left(Q_{N}^{ \pm}\right)^{*}$ whenever they follow the same marked pattern and the words $h_{N}\left(\zeta_{1}\right), h_{N}\left(\zeta_{2}\right)$ are freely irreducible.

Given a nonempty marked pattern $v \in\left(P_{N}^{ \pm}\right)^{*}$, let $v_{0}$ be the word obtained by deleting the last letter of $v$. For any $n \in N$ we substitute $a_{n}$ for each occurrence of $*_{n}$ in $v_{0}$ and $b_{n}^{-1}$ for each occurrence of $*_{n}^{-1}$. This yields a word $\eta \in\left(Q_{N}^{ \pm}\right)^{*}$ that follows the marked pattern $v_{0}$. Now let $\xi=\eta c_{n}$ if the last letter of $v$ is $*_{n}, n \in N$ and let $\xi=\eta c_{n}^{-1}$ if the last letter of $v$ is $*_{n}^{-1}$. Clearly, $\xi$ is a freely irreducible word following the marked pattern $v$. Moreover, $\xi \in\left(\widetilde{Q}_{N}^{ \pm}\right)^{*}$ and the word $h_{N}(\xi)$ is also freely irreducible.

We shall show that the set $Z_{N}(\xi)$ is contained in the orbit of $\xi$ under the $G\left(D^{(N)}\right)$ action on $\left(Q_{N}^{ \pm}\right)^{*}$. Take any $\zeta \in Z_{N}(\xi)$. If $\zeta$ is a word over the alphabet $\widetilde{Q}_{N}^{ \pm}$and $h_{N}(\zeta)$ is freely irreducible, then it follows from the above that $\zeta=g(\xi)$ for some $g \in \widetilde{G} \subset G\left(D^{(N)}\right)$. On the other hand, suppose that the last letter of $\zeta$ is $q_{n i}$ or $q_{n i}^{-1}$, where $n \in N, 1 \leq i \leq 2 n-2$. In this case we have $\zeta=\left(\pi_{\tau}^{(N)}\right)^{i}(\xi)$, where $\tau=\prod_{n \in N}\left(c_{n} q_{n 1} \ldots q_{n, 2 n-2}\right)$. By Proposition $4.4, \pi_{\tau}^{(N)} \in G\left(D^{(n)}\right)$.

It remains to consider the case when the last letter of $\zeta$ belongs to $\widetilde{Q}_{N}^{ \pm}$but the word $h_{N}(\zeta)$ is freely reducible. There is at most one $\zeta \in Z_{N}(\xi)$ with such properties. It exists if the last two letters of $v$ are $*_{l} *_{m}^{-1}$ or $*_{l}^{-1} *_{m}$, where $l, m \in N, l \neq m$. Assume this is the case. Then the last letter of the word $\eta$ is either $a_{l}$ or $b_{l}^{-1}$. Let us change this letter to $c_{l}$ or $c_{l}^{-1}$, respectively. The resulting word $\eta_{1}$ follows the marked pattern $v_{0}$. Also, the words $h_{N}(\eta)$ and $h_{N}\left(\eta_{1}\right)$ are freely irreducible. By Proposition 3.5, $h_{N}\left(\eta_{1}\right)=g_{1}\left(h_{N}(\eta)\right)$ for some $g_{1} \in G(D)$. There exists a unique $\zeta_{1} \in\left(\widetilde{Q}_{N}^{ \pm}\right)^{*}$ such that $h_{N}\left(\zeta_{1}\right)=g_{1}\left(h_{N}(\zeta)\right)$ and $v$ is the marked pattern of $\zeta_{1}$. By the above there exists $\tilde{g}_{1} \in \widetilde{G}$ such that $\tilde{g}_{1}(\eta)=\eta_{1}$ and $\tilde{g}_{1}(\zeta)=\zeta_{1}$. Since the word $h_{N}(\zeta)$ is freely reducible, so is $h_{N}\left(\zeta_{1}\right)$. On the other hand, the word $h_{N}\left(\eta_{1}\right)$, which can be obtained by deleting the last letter of $h_{N}\left(\zeta_{1}\right)$, is freely irreducible. It follows that the 
last two letters of $h_{N}\left(\zeta_{1}\right)$ are $c c^{-1}$ or $c^{-1} c$. Then the last two letters of $\zeta_{1}$ are $c_{l} c_{m}^{-1}$ or $c_{l}^{-1} c_{m}$. If $2 m-1$ does not divide $2 l-1$ then the word $\left(\pi_{\tau}^{(N)}\right)^{2 l-1}\left(\zeta_{1}\right)$ matches $\zeta_{1}$ except for the last letter. Consequently, the word $\zeta^{\prime}=\tilde{g}_{1}^{-1}\left(\pi_{\tau}^{(N)}\right)^{2 l-1} \tilde{g}_{1}(\zeta)$ matches $\zeta$ except for the last letter. Since the $G\left(D^{(N)}\right)$ action preserves marked patterns, the word $\zeta^{\prime}$ follows the marked pattern $v$. Hence $\zeta^{\prime} \in Z_{N}(\xi)$. As $\zeta^{\prime} \neq \zeta$, it follows from the above that $\zeta^{\prime}=g(\xi)$ for some $g \in G\left(D^{(N)}\right)$. Then $\zeta=g_{0}(\xi)$, where $g_{0}=\tilde{g}_{1}^{-1}\left(\pi_{\tau}^{(N)}\right)^{1-2 l} \tilde{g}_{1} g \in G\left(D^{(N)}\right)$.

Now suppose that $2 m-1$ divides $2 l-1$. Then $\left(\pi_{\tau}^{(N)}\right)^{2 l-1}\left(\zeta_{1}\right)=\zeta_{1}$ and the above argument does not apply. Recall that the last two letters of $h_{N}\left(\zeta_{1}\right)$ are $c c^{-1}$ or $c^{-1} c$. If these letters are preceded by $b^{-1}$, we let $\zeta_{2}=\pi_{\sigma_{1}}^{(N)}\left(\zeta_{1}\right)$. Otherwise they are preceded by $a$ or $h_{N}\left(\zeta_{1}\right)$ has length 2 . In this case, we let $\zeta_{2}=\zeta_{1}$. Further, consider the permutation $\tau_{1}=\tau^{2 m-1} \sigma_{2} \tau^{-(2 m-1)} \sigma_{2} \tau^{2 m-1}$ on $Q_{N}$. Since $\pi_{\tau}^{(N)}, \pi_{\sigma_{2}}^{(N)} \in G\left(D^{(n)}\right)$, we have that $\pi_{\tau_{1}}^{(N)}=\left(\pi_{\tau}^{(N)}\right)^{2 m-1} \pi_{\sigma_{2}}^{(N)}\left(\pi_{\tau}^{(N)}\right)^{1-2 m} \pi_{\sigma_{2}}^{(N)}\left(\pi_{\tau}^{(N)}\right)^{2 m-1} \in G\left(D^{(N)}\right)$. It is easy to see that $\tau_{1}\left(c_{m}\right)=c_{m}$ and $\tau_{1}\left(a_{n}\right)=a_{n}$ for all $n \in N$. Since $2 m-1<2 l-1$, we have $\tau_{1}\left(c_{l}\right)=b_{l}$. Also, for any $n \in N$ we have $\tau_{1}\left(b_{n}\right)=b_{n}$ if $2 n-1$ divides $2 m-1$ and $\tau_{1}\left(b_{n}\right)=c_{n}$ otherwise. It follows that $\zeta_{3}=\pi_{\tau_{1}}^{(N)}\left(\zeta_{2}\right)$ is a word in the alphabet $\widetilde{Q}_{N}^{ \pm}$such that $h_{N}\left(\zeta_{3}\right)$ is freely irreducible. Since $\zeta_{3}$ follows the marked pattern $v$, we obtain that $\zeta_{3}$ belongs to the orbit of $\xi$ under the $G\left(D^{(N)}\right)$ action. So does the word $\zeta$.

Proposition 4.7. Suppose that $\xi \in\left(Q_{N}^{ \pm}\right)^{*}$ is a freely irreducible word. Then the orbit of $\xi$ under the action of the group $G\left(D^{(N)}\right)$ on $\left(Q_{N}^{ \pm}\right)^{*}$ consists of all freely irreducible words following the same marked pattern as $\xi$.

Proof. First we shall show that the $G\left(D^{(N)}\right)$ action on $\left(Q_{N}^{ \pm}\right)^{*}$ preserves marked patterns and free irreducibility of words. Let $\phi_{N}^{ \pm}$and $\psi_{N}^{ \pm}$denote the state transition and output functions of the automaton $U^{(N)}$. By $\widetilde{\phi}_{N}$ and $\widetilde{\psi}_{N}$ denote the state transition and output functions of its dual $D^{(N)}$. Take any $q \in Q_{N}^{ \pm}$and $x \in X$. For every $n \in N$ we have that $\phi_{N}^{ \pm}(q, x) \in Q_{n}$ (resp. $\left.\phi_{N}^{ \pm}(q, x) \in Q_{n}^{ \pm}\right)$if and only if $q \in$ $Q_{n}$ (resp. $q \in Q_{n}^{ \pm}$). Since $\phi_{N}^{ \pm}(q, x)=\widetilde{\psi}_{N}(x, q)$, it follows that transformations $D_{0}^{(N)}$ and $D_{1}^{(N)}$ preserve marked patterns of words. So does any $g \in G\left(D^{(N)}\right)$. Further, let $p=\phi_{N}^{ \pm}(q, x)$ and $y=\psi_{N}^{ \pm}(q, x)$. Then $\phi_{N}^{ \pm}\left(q^{-1}, y\right)=p^{-1}$ and $\psi_{N}^{ \pm}\left(q^{-1}, y\right)=x$. Consequently, $D_{x}^{(N)}\left(q q^{-1}\right)=\tilde{\psi}_{N}(x, q) \tilde{\psi}_{N}\left(\tilde{\phi}_{N}(x, q), q^{-1}\right)=$ $\phi_{N}^{ \pm}(q, x) \phi_{N}^{ \pm}\left(q^{-1}, \psi_{N}^{ \pm}(q, x)\right)=p p^{-1}$. It follows that the set $P=\left\{q q^{-1} \mid q \in\right.$ $\left.Q_{N}^{ \pm}\right\} \subset\left(Q_{N}^{ \pm}\right)^{*}$ is invariant under $D_{0}^{(N)}$ and $D_{1}^{(N)}$. Any freely reducible word $\xi \in$ $\left(Q_{N}^{ \pm}\right)^{*}$ is represented as $\xi_{1} \xi_{0} \xi_{2}$, where $\xi_{0} \in P$ and $\xi_{1}, \xi_{2} \in\left(Q_{N}^{ \pm}\right)^{*}$. For any $x \in X$ we have $D_{x}^{(N)}(\xi)=D_{x}^{(N)}\left(\xi_{1}\right) D_{x_{0}}^{(N)}\left(\xi_{0}\right) D_{x_{1}}^{(N)}\left(\xi_{2}\right)$, where $x_{0}, x_{1} \in X$. By the above $D_{x}^{(N)}(\xi)$ is freely reducible. Thus $D_{0}^{(N)}$ and $D_{1}^{(N)}$ preserve free reducibility of words. Since these transformations are invertible, they also preserve free irreducibility, and so does any $g \in G\left(D^{(N)}\right)$. 
Now we are going to prove that for any freely irreducible words $\xi_{1}, \xi_{2} \in\left(Q_{N}^{ \pm}\right)^{*}$ following the same marked pattern $v$ there exists $g \in G\left(D^{(N)}\right)$ such that $\xi_{2}=g\left(\xi_{1}\right)$. The claim is proved by induction on the length of the marked pattern $v$. The empty marked pattern is followed only by the empty word. Now let $k \geq 1$ and assume that the claim holds for all marked patterns of length less than $k$. Take any marked pattern $v$ of length $k$. By Lemma 4.6, the marked pattern $v$ is followed by a freely irreducible word $\xi \in\left(Q_{N}^{ \pm}\right)^{*}$ such that the set $Z_{N}(\xi)$ is contained in an orbit of the $G\left(D^{(N)}\right)$ action. Suppose that $\xi_{1}, \xi_{2} \in\left(Q_{N}^{ \pm}\right)^{*}$ are freely irreducible words following the marked pattern $v$. Let $\eta, \eta_{1}, \eta_{2}$ be the words obtained by deleting the last letter of $\xi$, $\xi_{1}, \xi_{2}$, respectively. Then $\eta, \eta_{1}, \eta_{2}$ are freely irreducible and follow the same marked pattern of length $k-1$. By the inductive assumption there are $g_{1}, g_{2} \in G\left(D^{(N)}\right)$ such that $\eta=g_{1}\left(\eta_{1}\right)=g_{2}\left(\eta_{2}\right)$. Since the $G\left(D^{(N)}\right)$ action preserves marked patterns and free irreducibility, it follows that $g_{1}\left(\xi_{1}\right), g_{2}\left(\xi_{2}\right) \in Z_{N}(\xi)$. As $Z_{N}(\xi)$ is contained in an orbit, there exists $g_{0} \in G\left(D^{(N)}\right)$ such that $g_{0}\left(g_{1}\left(\xi_{1}\right)\right)=g_{2}\left(\xi_{2}\right)$. Then $\xi_{2}=g\left(\xi_{1}\right)$, where $g=g_{2}^{-1} g_{0} g_{1} \in G\left(D^{(N)}\right)$.

Corollary 4.8. Let $n$ be a positive integer. Suppose that $\xi \in\left(Q_{n}^{ \pm}\right)^{*}$ is a freely irreducible word. Then the orbit of $\xi$ under the action of the group $G\left(D^{(n)}\right)$ on $\left(Q_{n}^{ \pm}\right)^{*}$ consists of all freely irreducible words following the same pattern as $\xi$.

Corollary 4.9. The group defined by the dual automaton of an Aleshin type automaton $A^{(n)}$ acts transitively on each level of the rooted tree $Q_{n}^{*}$.

Corollary 4.8 is a particular case of Proposition 4.7, when $N=\{n\}$. Furthermore, Corollary 4.9 follows from Corollary 4.8 in the same way as Corollary 3.6 follows from Proposition 3.5. We omit the proof.

Theorem 4.10. For any nonempty set $N$ of positive integers the group $G\left(A^{(N)}\right)$ is the free non-Abelian group on generators $A_{q}^{(N)}, q \in Q_{N}$.

Proof. The group $G\left(A^{(N)}\right)$ is the free non-Abelian group on generators $A_{q}, q \in$ $Q_{N}$, if and only if $\left(A_{q_{1}}^{(N)}\right)^{m_{1}}\left(A_{q_{2}}^{(N)}\right)^{m_{2}} \ldots\left(A_{q_{k}}^{(N)}\right)^{m_{k}} \neq 1$ for any pair of sequences $q_{1}, \ldots, q_{k}$ and $m_{1}, \ldots, m_{k}$ such that $k>0, q_{i} \in Q_{N}$ and $m_{i} \in \mathbb{Z} \backslash\{0\}$ for $1 \leq i \leq k$, and $q_{i} \neq q_{i+1}$ for $1 \leq i \leq k-1$. Since $U_{q}^{(N)}=A_{q}^{(N)}$ and $U_{q^{-1}}^{(N)}=\left(A_{q}^{(N)}\right)^{-1}$ for all $q \in Q_{N}$, an equivalent condition is that $U_{\xi}^{(N)} \neq 1$ for any nonempty freely irreducible word $\xi \in\left(Q_{N}^{ \pm}\right)^{*}$.

Suppose that $U_{\xi}^{(N)}=1$ for some freely irreducible word $\xi \in\left(Q_{N}^{ \pm}\right)^{*}$. By Corollary 2.4, $U_{g(\xi)}^{(N)}=1$ for all $g \in S\left(D^{(N)}\right)$. Since the transformation semigroup $S\left(D^{(N)}\right)$ has the same orbits as the transformation group $G\left(D^{(N)}\right)$, it follows that $U_{g(\xi)}^{(N)}=1$ for all $g \in G\left(D^{(N)}\right)$. Now Proposition 4.7 implies that $U_{\eta}^{(N)}=1$ for any freely irreducible word $\eta \in\left(Q_{N}^{ \pm}\right)^{*}$ following the same marked pattern as $\xi$. 
In particular, $U_{\eta}^{(N)}$ acts trivially on the first level of the rooted binary tree $\{0,1\}^{*}$. Finally, Lemma 4.5 implies that $\xi$ follows the empty marked pattern. Then $\xi$ itself is the empty word.

\section{The Bellaterra automaton and its series}

In this section we consider the Bellaterra automaton, a series of automata of Bellaterra type, and their disjoint unions. We use the notation of Sections 3 and 4.

The Bellaterra automaton $B$ is an automaton over the alphabet $X=\{0,1\}$ with the set of internal states $Q=\{a, b, c\}$. The state transition function $\widehat{\phi}$ and the output function $\hat{\psi}$ of $B$ are defined as follows: $\hat{\phi}(a, 0)=\widehat{\phi}(b, 1)=c, \hat{\phi}(a, 1)=\hat{\phi}(b, 0)=$ $b, \hat{\phi}(c, 0)=\widehat{\phi}(c, 1)=a ; \hat{\psi}(a, 0)=\hat{\psi}(b, 0)=\widehat{\psi}(c, 1)=0, \widehat{\psi}(a, 1)=\widehat{\psi}(b, 1)=$ $\hat{\psi}(c, 0)=1$. The Moore diagram of $B$ is depicted in Figure 2. It is easy to verify that the inverse automaton of $B$ coincides with $B$. Besides, the reverse automaton of $B$ can be obtained from $B$ by renaming its states $a$ and $c$ to $c$ and $a$, respectively Lemma 2.5 implies that $B$ is bi-reversible.

The Bellaterra automaton $B$ is closely related to the Aleshin automaton $A$. Namely, the two automata share the alphabet, the set of internal states, and the state transition function. On the other hand, the output function $\hat{\psi}$ of $B$ never coincides with the output function $\psi$ of $A$, that is, $\hat{\psi}(q, x) \neq \psi(q, x)$ for all $q \in Q$ and $x \in X$.

For any integer $n \geq 1$ we define a Bellaterra type automaton $B^{(n)}$ as the automaton that is related to the Aleshin type automaton $A^{(n)}$ in the same way as the automaton $B$ is related to $A$. To be precise, $B^{(n)}$ is an automaton over the alphabet $X=\{0,1\}$ with the set of states $Q_{n}$. The state transition function of $B^{(n)}$ coincides with that of $A^{(n)}$. The output function $\hat{\psi}_{n}$ of $B^{(n)}$ is defined so that for any $x \in X$ we have $\widehat{\psi}_{n}(q, x)=x$ if $q \in\left\{a_{n}, b_{n}\right\}$ and $\hat{\psi}_{n}(q, x)=1-x$ if $q \in Q_{n} \backslash\left\{a_{n}, b_{n}\right\}$. Then $\widehat{\psi}_{n}(q, x)=1-\psi_{n}(q, x)$ for all $q \in Q_{n}$ and $x \in X$, where $\psi_{n}$ is the output function of $A^{(n)}$. Note that the automaton $B^{(1)}$ coincides with $B$ up to renaming of the internal states.

In addition, we define a Bellaterra type automaton $B^{(0)}$. This is an automaton over the alphabet $X$ with the set of internal states $Q_{0}$ consisting of a single element $c_{0}$. The state transition function $\hat{\phi}_{0}$ and the output function $\hat{\psi}_{0}$ of $B^{(0)}$ are defined as follows: $\widehat{\phi}_{0}\left(c_{0}, 0\right)=\widehat{\phi}_{0}\left(c_{0}, 1\right)=c_{0} ; \widehat{\psi}_{0}\left(c_{0}, 0\right)=1, \widehat{\psi}_{0}\left(c_{0}, 1\right)=0$.

It is easy to see that each Bellaterra type automaton $B^{(n)}$ coincides with its inverse automaton. The reverse automaton of $B^{(0)}$ coincides with $B^{(0)}$ as well. In the case $n \geq 1$, the reverse automaton of $B^{(n)}$ can be obtained from $B^{(n)}$ by renaming its states $c_{n}, q_{n 1}, \ldots, q_{n, 2 n-2}, a_{n}$ to $a_{n}, q_{n, 2 n-2}, \ldots, q_{n 1}, c_{n}$, respectively. Lemma 2.5 implies that each $B^{(n)}$ is bi-reversible.

Let $N$ be a nonempty finite set of nonnegative integers. We denote by $B^{(N)}$ the disjoint union of automata $B^{(n)}, n \in N$. Then $B^{(N)}$ is an automaton over the alphabet $X=\{0,1\}$ with the set of internal states $Q_{N}=\bigcup_{n \in N} Q_{n}$. It is bi-reversible since 

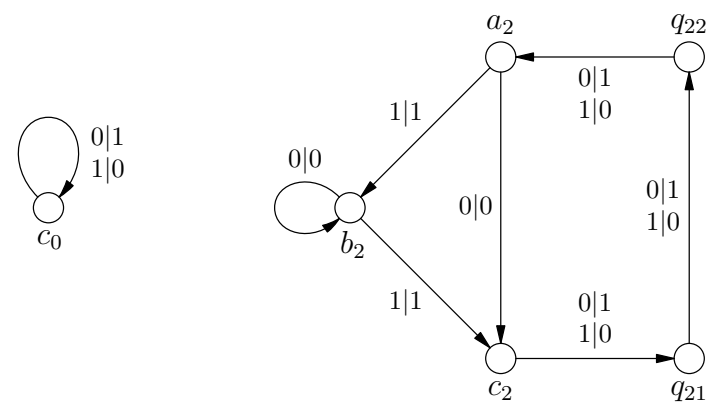

Figure 7. Automaton $B^{(\{0,2\})}$.

each $B^{(n)}$ is bi-reversible. If $0 \notin N$, then the automaton $B^{(N)}$ shares its alphabet, its internal states, and its state transition function with the automaton $A^{(N)}$ while the output functions of these automata never coincide.

The relation between automata of Aleshin type and of Bellaterra type induces a relation between transformations defined by automata of these two types.

Lemma 5.1. Let $h=B_{c_{0}}^{(0)}$. Then

(i) $A_{q}=h B_{q}$ and $B_{q}=h A_{q}$ for any $q \in\{a, b, c\}$;

(ii) $A_{q}^{(n)}=h B_{q}^{(n)}$ and $B_{q}^{(n)}=h A_{q}^{(n)}$ for any $n \geq 1$ and $q \in Q_{n}$;

(iii) $A_{q}^{(N)}=h B_{q}^{(N)}$ and $B_{q}^{(N)}=h A_{q}^{(N)}$ for any nonempty finite set $N$ of positive integers and any $q \in Q_{N}$.

Proof. The transformation $h$ is the automorphism of the free monoid $\{0,1\}^{*}$ that interchanges the free generators 0 and 1. For any $w \in X^{*}$ the word $h(w)$ can be obtained from $w$ by changing all letters 0 to 1 and all letters 1 to 0 .

Suppose that $\widetilde{A}$ and $\widetilde{B}$ are two automata over the alphabet $X$ such that their sets of internal states and state transition functions are the same but their output functions never coincide. It is easy to see that $\widetilde{A}_{q}=h \widetilde{B}_{q}$ and $\widetilde{B}_{q}=h \widetilde{A}_{q}$ for any internal state $q$ of the automata $\widetilde{A}$ and $\widetilde{B}$. The lemma follows.

Proposition 5.2. (i) The group $G(A)$ is an index 2 subgroup of $G\left(B^{(\{0,1\})}\right)$.

(ii) For any $n \geq 1$ the group $G\left(A^{(n)}\right)$ is an index 2 subgroup of $G\left(B^{(\{0, n\})}\right)$.

(iii) For any nonempty finite set $N$ of positive integers the group $G\left(A^{(N)}\right)$ is an index 2 subgroup of $G\left(B^{(N \cup\{0\})}\right)$.

Proof. Note that the statement (i) is a particular case of the statement (ii) as $G(A)=$ $G\left(A^{(1)}\right)$. Furthermore, the statement (ii) is a particular case of the statement (iii) since $A^{(n)}=A^{(\{n\})}$ for any integer $n \geq 1$.

Let $N$ be a nonempty finite set of positive integers. The group $G\left(A^{(N)}\right)$ is generated by transformations $A_{q}^{(N)}, q \in Q_{N}$. The group $G\left(B^{(N \cup\{0\})}\right)$ is generated by 
transformations $h=B_{c_{0}}^{(0)}$ and $B_{q}^{(N)}, q \in Q_{N}$. By Lemma 5.1, $A_{q}^{(N)}=h B_{q}^{(N)}$ and $B_{q}^{(N)}=h A_{q}^{(N)}$ for any $q \in Q_{N}$. It follows that the group $G\left(B^{(N \cup\{0\})}\right)$ is generated by transformations $h$ and $A_{q}^{(N)}, q \in Q_{N}$. In particular, $G\left(A^{(N)}\right) \subset G\left(B^{(N \cup\{0\})}\right)$.

For any $n \geq 0$ the automaton $B^{(n)}$ coincides with its inverse. Lemma 2.1 implies that $h^{2}=1$ and $\left(B_{q}^{(N)}\right)^{2}=1, q \in Q_{N}$. Then $h A_{q}^{(N)} h^{-1}=B_{q}^{(N)} h=\left(A_{q}^{(N)}\right)^{-1}$ for any $q \in Q_{N}$. It follows that $G\left(A^{(N)}\right)$ is a normal subgroup of $G\left(B^{(N \cup\{0\})}\right)$. Since $h^{2}=1$, the index of the group $G\left(A^{(N)}\right)$ in $G\left(B^{(N \cup\{0\})}\right)$ is at most 2 . On the other hand, $G\left(A^{(N)}\right) \neq G\left(B^{(N \cup\{0\})}\right)$ as $G\left(B^{(N \cup\{0\})}\right)$ contains a nontrivial involution $h$, while $G\left(A^{(N)}\right)$ is a free group due to Theorem 4.10. Thus $G\left(A^{(N)}\right)$ is an index 2 subgroup of $G\left(B^{(N \cup\{0\})}\right)$.

The relation between groups defined by automata of Aleshin type and of Bellaterra type allows us to establish the structure of the groups defined by automata of the latter type. As the following two theorems show, these groups are free products of groups of order 2.

Theorem 5.3 ([Nek]). The group $G(B)$ is freely generated by involutions $B_{a}, B_{b}, B_{c}$.

Theorem 5.4. (i) For any $n \geq 1$ the group $G\left(B^{(n)}\right)$ is freely generated by $2 n+1$ involutions $B_{q}^{(n)}, q \in Q_{n}$.

(ii) For any nonempty finite set $N$ of nonnegative integers the group $G\left(B^{(N)}\right)$ is freely generated by involutions $B_{q}^{(N)}, q \in Q_{N}$.

To prove Theorems 5.3 and 5.4, we need the following lemma.

Lemma 5.5. Suppose that a group $G$ is generated by elements $g_{0}, g_{1}, \ldots, g_{k}(k \geq 1)$ of order at most 2. Let $H$ be the subgroup of $G$ generated by elements $h_{i}=g_{0} g_{i}$, $1 \leq i \leq k$. Then $G$ is freely generated by $k+1$ involutions $g_{0}, g_{1}, \ldots, g_{k}$ if and only if $H$ is the free group on $k$ generators $h_{1}, \ldots, h_{k}$.

Proof. Consider an element $h=h_{i_{1}}^{\varepsilon_{1}} h_{i_{2}}^{\varepsilon_{2}} \ldots h_{i_{l}}^{\varepsilon_{l}}$, where $l \geq 1,1 \leq i_{j} \leq k, \varepsilon_{j} \in$ $\{-1,1\}$, and $\varepsilon_{j}=\varepsilon_{j+1}$ whenever $i_{j}=i_{j+1}$. Since $h_{i}=g_{0} g_{i}$ and $h_{i}^{-1}=g_{i} g_{0}$ for $1 \leq i \leq k$, and $g_{0}^{2}=1$, we obtain that $h=g_{0}^{\prime} g_{i_{1}} g_{1}^{\prime} \ldots g_{i_{l}} g_{l}^{\prime}$, where each $g_{j}^{\prime}$ is equal to $g_{0}$ or 1 . Moreover, $g_{j}^{\prime}=g_{0}$ whenever $\varepsilon_{j}=\varepsilon_{j+1}$. In particular, $h \neq 1$ if $G$ is freely generated by involutions $g_{0}, g_{1}, \ldots, g_{k}$. It follows that $H$ is the free group on generators $h_{1}, \ldots, h_{k}$ if $G$ is freely generated by involutions $g_{0}, g_{1}, \ldots, g_{k}$.

Now assume that $H$ is the free group on generators $h_{1}, \ldots, h_{k}$. Then each $h_{i}$ has infinite order. Since $h_{i}=g_{0} g_{i}$ and $g_{0}^{2}=g_{i}^{2}=1$, it follows that $g_{0} \neq 1$ and $g_{i} \neq 1$. Hence each of the elements $g_{0}, g_{1}, \ldots, g_{k}$ has order 2. In particular, none of these elements belongs to the free group $H$.

The group $G$ is freely generated by involutions $g_{0}, g_{1}, \ldots, g_{k}$ if $g \neq 1$ for any $g=g_{i_{1}} \ldots g_{i_{l}}$ such that $l \geq 1,0 \leq i_{j} \leq k$, and $i_{j} \neq i_{j+1}$. First consider the 
case when $l$ is even. Note that $g_{i} g_{j}=h_{i}^{-1} h_{j}$ for $0 \leq i, j \leq n$, where by definition $h_{0}=1$. Therefore $g=h_{i_{1}}^{-1} h_{i_{2}} \ldots h_{i_{l-1}}^{-1} h_{i_{l}} \in H$. Since $h_{0}=1$, the sequence $h_{i_{1}}^{-1}, h_{i_{2}}, \ldots, h_{i_{l-1}}^{-1}, h_{i_{l}}$ can contain the unit elements. After removing all of them, we obtain a nonempty sequence in which neighboring elements are not inverses of each other. Since $h_{1}, \ldots, h_{k}$ are free generators, we conclude that $g \neq 1$. In the case when $l$ is odd, it follows from the above that $g=g_{i_{1}} h$, where $h \in H$. Since $g_{i_{1}} \notin H$, we have that $g \notin H$, in particular, $g \neq 1$.

Proof of Theorem 5.3 and Theorem 5.4. First we observe that Theorem 5.3 is a particular case of Theorem 5.4 since the automata $B$ and $B^{(1)}$ coincide up to renaming of their internal states. Further, the statement (i) of Theorem 5.4 is a particular case of the statement (ii) since $B^{(n)}=B^{(\{n\})}$ for any $n \geq 1$.

Suppose that $N$ is a nonempty finite set of nonnegative integers such that $0 \in N$. For any $n \in N$ the automaton $B^{(n)}$ coincides with its inverse. Lemma 2.1 implies that $\left(B_{q}^{(N)}\right)^{2}=1$ for all $q \in Q_{N}$. If $N=\{0\}$ then $Q_{N}=\left\{c_{0}\right\}$ and $G\left(B^{(N)}\right)$ is a group of order 2 generated by the involution $h=B_{c_{0}}^{(0)}$. Now assume that $N \neq\{0\}$. Then $K=N \backslash\{0\}$ is a nonempty set of positive integers. The group $G\left(B^{(N)}\right)$ is generated by transformations $h$ and $B_{q}^{(K)}, q \in Q_{K}$. All generators are of order at most 2. The group $G\left(A^{(K)}\right)$ is the free group on generators $A_{q}^{(K)}, q \in Q_{K}$ due to Theorem 4.10. By Lemma 5.1, $A_{q}^{(K)}=h B_{q}^{(K)}$ for any $q \in Q_{K}$. Then Lemma 5.5 implies that $G\left(B^{(N)}\right)$ is freely generated by involutions $h$ and $B_{q}^{(K)}, q \in Q_{K}$.

Now consider the case when $N$ is a nonempty finite set of positive integers. By the above the group $G\left(B^{(N \cup\{0\})}\right)$ is freely generated by involutions $h$ and $B_{q}^{(N)}, q \in Q_{N}$. Clearly, this implies that the group $G\left(B^{(N)}\right)$ is freely generated by involutions $B_{q}^{(N)}$, $q \in Q_{N}$.

Now we shall establish a relation between transformation groups defined by the Aleshin type and the Bellaterra type automata with the same set of internal states.

Since $G(A)$ is the free group on generators $A_{a}, A_{b}, A_{c}$, there is a unique homomorphism $\Delta: G(A) \rightarrow G(B)$ such that $\Delta\left(A_{a}\right)=B_{a}, \Delta\left(A_{b}\right)=B_{b}, \Delta\left(A_{c}\right)=B_{c}$. Likewise, for any $n \geq 1$ there is a unique homomorphism $\Delta_{n}: G\left(A^{(n)}\right) \rightarrow G\left(B^{(n)}\right)$ such that $\Delta_{n}\left(A_{q}^{(n)}\right)=B_{q}^{(n)}$ for all $q \in Q_{n}$. Also, for any nonempty finite set $N$ of positive integers there is a unique homomorphism $\Delta_{N}: G\left(A^{(N)}\right) \rightarrow G\left(B^{(N)}\right)$ such that $\Delta_{N}\left(A_{q}^{(N)}\right)=B_{q}^{(N)}$ for all $q \in Q_{N}$.

Proposition 5.6. (i) $G(A) \cap G(B)=\{g \in G(A) \mid \Delta(g)=g\}$.

(ii) $G(A) \cap G(B)$ is the free group on generators $B_{a} B_{b}$ and $B_{a} B_{c}$.

(iii) $G(A) \cap G(B)$ is an index 2 subgroup of $G(B)$.

(iv) $A_{p}^{-1} A_{q}=B_{p} B_{q}$ for all $p, q \in\{a, b, c\}$.

Proof. Let $h=B_{c_{0}}^{(0)}$. By Lemma 5.1, $A_{q}=h B_{q}$ for all $q \in\{a, b, c\}$. Since the inverse automaton of $B$ coincides with $B$, Lemma 2.1 implies that $B_{a}^{2}=B_{b}^{2}=$ 
$B_{c}^{2}=1$. Then for any $p, q \in\{a, b, c\}$ we have $A_{p}^{-1} A_{q}=\left(h B_{p}\right)^{-1} h B_{q}=B_{p}^{-1} B_{q}=$ $B_{p} B_{q}$.

It is easy to see that $\{g \in G(A) \mid \Delta(g)=g\}$ is a subgroup of $G(A) \cap G(B)$. Let $\widetilde{G}$ be the group generated by transformations $B_{a} B_{b}$ and $B_{a} B_{c}$. By the above $\Delta\left(A_{a}^{-1} A_{b}\right)=B_{a}^{-1} B_{b}=B_{a} B_{b}=A_{a}^{-1} A_{b}$ and $\Delta\left(A_{a}^{-1} A_{c}\right)=B_{a}^{-1} B_{c}=B_{a} B_{c}=$ $A_{a}^{-1} A_{c}$. It follows that $\widetilde{G}$ is a subgroup of $\{g \in G(A) \mid \Delta(g)=g\}$.

By Theorem 5.3, the group $G(B)$ is freely generated by involutions $B_{a}, B_{b}$, $B_{c}$. Then Lemma 5.5 implies that $\widetilde{G}$ is the free group on generators $B_{a} B_{b}$ and $B_{a} B_{c}$. Note that $B_{a} B_{q} \in \widetilde{G}$ for all $q \in Q$. Then for any $p, q \in Q$ we have $B_{p} B_{q}=\left(B_{a} B_{p}\right)^{-1} B_{a} B_{q} \in \widetilde{G}$. It follows that for any $g \in G(B)$ at least one of the transformations $g$ and $B_{a} g$ belongs to $\widetilde{G}$. Therefore the index of $\widetilde{G}$ in $G(B)$ is at most 2.

Note that $B_{a} \notin G(A)$ as $B_{a}$ is a nontrivial involution while $G(A)$ is a free group. Hence $G(A) \cap G(B) \neq G(B)$. Now it follows from the above that $\widetilde{G}=\{g \in G(A) \mid$ $\Delta(g)=g\}=G(A) \cap G(B)$ and this is an index 2 subgroup of $G(B)$.

Proposition 5.7. Let $n$ be a positive integer. Then

(i) $G\left(A^{(n)}\right) \cap G\left(B^{(n)}\right)=\left\{g \in G\left(A^{(n)}\right) \mid \Delta_{n}(g)=g\right\}$;

(ii) $G\left(A^{(n)}\right) \cap G\left(B^{(n)}\right)$ is the free group on $2 n$ generators $B_{a_{n}}^{(n)} B_{q}^{(n)}, q \in Q_{n} \backslash\left\{a_{n}\right\}$;

(iii) $G\left(A^{(n)}\right) \cap G\left(B^{(n)}\right)$ is an index 2 subgroup of $G\left(B^{(n)}\right)$;

(iv) $\left(A_{p}^{(n)}\right)^{-1} A_{q}^{(n)}=B_{p}^{(n)} B_{q}^{(n)}$ for all $p, q \in Q_{n}$.

Proposition 5.8. Let $N$ be a nonempty finite set of positive integers. Then

(i) $G\left(A^{(N)}\right) \cap G\left(B^{(N)}\right)=\left\{g \in G\left(A^{(N)}\right) \mid \Delta_{N}(g)=g\right\}$;

(ii) for any $n \in N$ the group $G\left(A^{(N)}\right) \cap G\left(B^{(N)}\right)$ is the free group on generators $B_{a_{n}}^{(N)} B_{q}^{(N)}, q \in Q_{N} \backslash\left\{a_{n}\right\}$

(iii) $G\left(A^{(N)}\right) \cap G\left(B^{(N)}\right)$ is an index 2 subgroup of $G\left(B^{(N)}\right)$;

(iv) $\left(A_{p}^{(N)}\right)^{-1} A_{q}^{(N)}=B_{p}^{(N)} B_{q}^{(N)}$ for all $p, q \in Q_{N}$.

The proofs of Propositions 5.7 and 5.8 are completely analogous to the proof of Proposition 5.6 and we omit them.

Now let us consider the dual automata of the Bellaterra automaton and automata of Bellaterra type.

Let $\hat{D}$ denote the dual automaton of the Bellaterra automaton $B$. The automaton $\widehat{D}$ is defined over the alphabet $Q=\{a, b, c\}$, with two internal states 0 and 1 . The Moore diagram of $\hat{D}$ is depicted in Figure 8. The automaton $\hat{D}$ is bi-reversible since $B$ is bi-reversible.

A word $\xi$ over an arbitrary alphabet is called a double letter word if there are two adjacent letters in $\xi$ that coincide. Otherwise we call $\xi$ a no-double-letter word. The set of no-double-letter words over the alphabet $Q$ forms a subtree of the rooted ternary tree $Q^{*}$. As an unrooted tree, this subtree is 3-regular. However it is not 


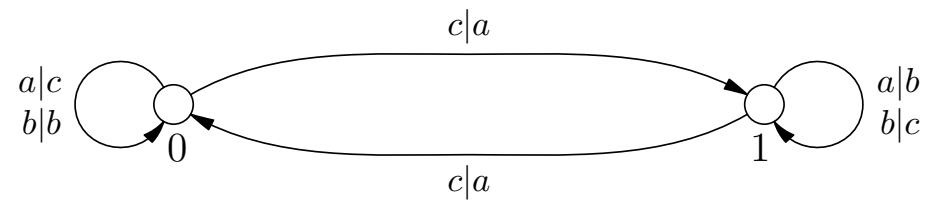

Figure 8. The dual automaton $\widehat{D}$.

regular as a rooted tree. The following proposition shows that the group $G(\hat{D})$ acts transitively on each level of the subtree.

Proposition 5.9 ([Nek]). Suppose that $\xi \in Q^{*}$ is a no-double-letter word. Then the orbit of $\xi$ under the action of the group $G(\widehat{D})$ on $Q^{*}$ consists of all no-double-letter words of the same length as $\xi$.

Proof. Let $\lambda$ and $\mu$ denote the state transition and output functions of the automaton $B$. By $\tilde{\lambda}$ and $\tilde{\mu}$ denote the state transition and output functions of its dual $\hat{D}$. Take any $q \in Q$ and $x \in X$. Let $p=\lambda(q, x)$ and $y=\mu(q, x)$. Since $B$ coincides with its inverse automaton, it follows that $p=\lambda(q, y)$. Consequently, $\widehat{D}_{x}(q q)=$ $\tilde{\mu}(x, q) \tilde{\mu}(\tilde{\lambda}(x, q), q)=\lambda(q, x) \lambda(q, \mu(q, x))=p p$. It follows that the set $P=$ $\{q q \mid q \in Q\} \subset Q^{*}$ is invariant under $\hat{D}_{0}$ and $\widehat{D}_{1}$. Any double letter word $\xi \in Q^{*}$ is represented as $\xi_{1} \xi_{0} \xi_{2}$, where $\xi_{0} \in P$ and $\xi_{1}, \xi_{2} \in Q^{*}$. For any $x \in X$ we have $\hat{D}_{x}(\xi)=\hat{D}_{x}\left(\xi_{1}\right) \hat{D}_{x_{0}}\left(\xi_{0}\right) \hat{D}_{x_{1}}\left(\xi_{2}\right)$, where $x_{0}, x_{1} \in X$. By the above $\hat{D}_{x}(\xi)$ is a double letter word. Thus $\widehat{D}_{0}$ and $\widehat{D}_{1}$ map double letter words to double letter words. Since these transformations are invertible, they also map no-double-letter words to no-double-letter words, and so does any $g \in G(\widehat{D})$.

Now we are going to prove that for any no-double-letter words $\xi_{1}, \xi_{2} \in Q^{*}$ of the same length $l$ there exists $g \in G(\widehat{D})$ such that $\xi_{2}=g\left(\xi_{1}\right)$. The empty word is the only word of length 0 so it is no loss to assume that $l>0$. First consider the case when $l$ is even. We have $\xi_{1}=q_{1} q_{2} \ldots q_{l-1} q_{l}$ and $\xi_{2}=p_{1} p_{2} \ldots p_{l-1} p_{l}$ for some $q_{i}, p_{i} \in Q, 1 \leq i \leq l$. Consider two words $\eta_{1}=q_{1} q_{2}^{-1} \ldots q_{l-1} q_{l}^{-1}$ and $\eta_{2}=p_{1} p_{2}^{-1} \ldots p_{l-1} p_{l}^{-1}$ over the alphabet $Q^{ \pm}$. Clearly, $\eta_{1}$ and $\eta_{2}$ follow the same pattern. Furthermore, they are freely irreducible since $\xi_{1}$ and $\xi_{2}$ are no-double-letter words. By Proposition 3.5, $\eta_{2}=g_{0}\left(\eta_{1}\right)$ for some $g_{0} \in G(D)$ (recall that $D$ is the dual of the automaton $U$, which is the disjoint union of the Aleshin automaton $A$ and its inverse). Since the $G(D)$ action on $\left(Q^{ \pm}\right)^{*}$ has the same orbits as the action of the semigroup $S(D)$, we may assume that $g_{0} \in S(D)$. Then $g_{0}=D_{w}$ for some word $w \in X^{*}$. Proposition 2.3 implies that $U_{\eta_{1}}(w u)=U_{\eta_{1}}(w) U_{\eta_{2}}(u)$ for any $u \in X^{*}$. By Proposition 5.6, $A_{p}^{-1} A_{q}=B_{p} B_{q}$ for all $p, q \in Q$. It follows that $U_{\eta_{1}}=B_{\xi_{1}}$ and $U_{\eta_{2}}=B_{\xi_{2}}$. In particular, $B_{\xi_{1}}(w u)=B_{\xi_{1}}(w) B_{\xi_{2}}(u)$ for any $u \in X^{*}$. Now Proposition 2.3 implies that $B_{\xi_{2}}=B_{g\left(\xi_{1}\right)}$, where $g=\widehat{D}_{w} \in G(\hat{D})$. By the above $g\left(\xi_{1}\right)$ is a no-double-letter word. By Theorem 5.3, the group $G(B)$ is freely generated by involutions $B_{q}, q \in Q$. Since $\xi_{2}$ and $g\left(\xi_{1}\right)$ are no-double-letter 
words in the alphabet $Q$, the equality $B_{\xi_{2}}=B_{g\left(\xi_{1}\right)}$ implies that $\xi_{2}=g\left(\xi_{1}\right)$.

Now consider the case when $\xi_{1}$ and $\xi_{2}$ have odd length. Obviously, there exist letters $q_{0}, p_{0} \in Q$ such that $\xi_{1} q_{0}$ and $\xi_{2} p_{0}$ are no-double-letter words. Since $\xi_{1} q_{0}$ and $\xi_{2} p_{0}$ are of the same even length, it follows from the above that $\xi_{2} p_{0}=g\left(\xi_{1} q_{0}\right)$ for some $g \in G(\hat{D})$. Then $\xi_{2}=g\left(\xi_{1}\right)$.

For any integer $n \geq 0$ let $\widehat{D}^{(n)}$ denote the dual automaton of the automaton $B^{(n)}$. The automaton $\widehat{D}^{(n)}$ is defined over the alphabet $Q_{n}$, with two internal states 0 and 1 . It is bi-reversible since $B^{(n)}$ is bi-reversible.

Proposition 5.10. Let $n \geq 1$ and suppose that $\xi \in Q_{n}^{*}$ is a no-double-letter word. Then the orbit of $\xi$ under the action of the group $G\left(\widehat{D}^{(n)}\right)$ on $Q_{n}^{*}$ consists of all no-double-letter words of the same length as $\xi$.

The proof of Proposition 5.10 is completely analogous to the above proof of Proposition 5.9 and we omit it.

\section{References}

[Ale] S. V. Aleshin, A free group of finite automata. Vestnik Moskov. Univ. Ser. I Mat. Mekh. 1983 (1983), no. 4, 12-14; English transl. Mosc. Univ. Math. Bull. 38 (1983), no. 4, 10-13. Zbl 0513.68044 MR 713968 378, 390

[BGN] L. Bartholdi, R. Grigorchuk, and V. Nekrashevych, From fractal groups to fractal sets. In Fractals in Graz 2001, Trends Math., Birkhäuser, Basel 2003, 25-118. Zbl 1037.20040 MR 2091700 378

[BGS] L. Bartholdi, R. I. Grigorchuk, and Z. Šunik, Branch groups. In Handbook of algebra. Vol. 3, North-Holland, Amsterdam 2003, 989-1112. Zbl 02156384 MR 2035113378

[BS] A. M. Brunner and S. Sidki, The generation of $\operatorname{GL}(n, \mathbb{Z})$ by finite state automata. Internat. J. Algebra Comput. 8 (1998), 127-139. Zbl 0923.20023 MR 1492064378

[GM] Y. Glasner and S. Mozes, Automata and square complexes. Geom. Dedicata 111 (2005), 43-64. Zbl 1088.20037 MR 2155175378

[Gri] R. I. Grigorchuk, Cancellative semigroups of power growth. Mat. Zametki 43 (1988), 305-319; English transl. Math. Notes 43 (1988), 175-183. Zbl 0643.20036 MR 941053378

[GNS] R. I. Grigorchuk, V. V. Nekrashevich, and V. I. Sushchanskii, Automata, dynamical systems, and groups. Trudy Mat. Inst. Steklov. 231 (2000), 134-214; English transl. Proc. Steklov Inst. Math. 2000 (2000), no. 4, 128-203. Zbl 01729301 MR 1841755 377, 384

[Hoř] J. Hořejš, Transformations defined by finite automata. Probl. Kibernetiki 9 (1963), 23-26; German transl. Probl. Kybernetik 6 (1966) , 31-35. Zbl 0178.32903378

[MNS] O. Macedońska, V. Nekrashevych, and V. Sushchansky, Commensurators of groups and reversible automata. Dopov. Nats. Akad. Nauk Ukr. Mat. Prirodozn. Tekh. Nauki 2000 (2000), no. 12, 36-39. Zbl 0977.20022 MR 1841119378 
[Nek] V. Nekrashevych, Self-similar groups. Math. Surveys Monogr. 117, Amer. Math. Soc., Providence, RI, 2005. Zbl 1087.20032 MR 2162164 378, 380, 382, 385, 400, 403

[Oli1] A. S. Olijnyk, Free groups of automaton permutations. (Ukrainian). Dopov. Nats. Akad. Nauk Ukr. Mat. Prirodozn. Tekh. Nauki 1998 (1998), no. 7, 40-44. Zbl 0933.20011 MR 1698343378

[Oli2] A. S. Olijnyk, Free products of finite groups and groups of finitely automatic permutations. Trudy Mat. Inst. Steklov. 231 (2000), 323-331; Proc. Steklov Inst. Math. 2000 (2000), no. 4, 308-315. Zbl 1005.20023 MR 1841761378

[Sid] S. Sidki, Automorphisms of one-rooted trees: growth, circuit structure, and acyclicity. J. Math. Sci. 100 (2000), 1925-1943. Zbl 1069.20504 MR 1774362379

[VV] M. Vorobets and Y. Vorobets, On a free group of transformations defined by an automaton. Geom. Dedicata 124 (2007), 237-249. Zbl 05180047 MR 2318547 379, $382,385,387,388,389,390$

Received April 29, 2006; revised September 15, 2008

M. Vorobets, Y. Vorobets, Department of Mathematics, Texas A\&M University, College Station, TX 77843-3368, U.S.A.

E-mail: mvorobet@math.tamu.edu; yvorobet@math.tamu.edu 